\title{
Treatment with lentiviral vectors encoding shRNA against interleukin 10 modulates the immunosuppressive activity of murine colon carcinoma-associated myeloid-derived suppressor cells
}

\author{
NATALIA ANGER-GÓRA, KATARZYNA WĘGIEREK-CIURA, AGNIESZKA SZCZYGIEŁ, \\ JAGODA MIERZEJEWSKA, ELŻBIETA PAJTASZ-PIASECKA and JOANNA ROSSOWSKA \\ Hirszfeld Institute of Immunology and Experimental Therapy, \\ Polish Academy of Sciences, 53-114 Wrocław, Poland
}

Received December 20, 2020; Accepted March 23, 2021

DOI: $10.3892 / \mathrm{ol} .2021 .12843$

\begin{abstract}
Myeloid-derived suppressor cells (MDSCs) are potent suppressors of antitumor immunity and their accumulation is often associated with poor prognosis. The aim of the present study was to determine the mechanisms of action of lentiviral vectors encoding short hairpin (sh)RNA against interleukin-10 (IL-10), with particular emphasis on their influence on the activity of tumor-derived MDSCs. Lentiviral vectors encoding shRNA against IL-10 (shIL-10 LVs) were utilized to silence the expression of IL-10 either in MDSCs that were generated ex vivo from bone marrow cells cultured in the presence of supernatant from MC38 colon carcinoma cells, or in situ in the MC38 murine colon carcinoma environment. Although monocytic MDSCs (M-MDSCs) transduced with shIL-10 LVs exhibited increased suppressor activity, transduction of polymorphonuclear MDSCs (PMN-MDSCs) appeared to reduce their ability to inhibit T lymphocyte functions. Analysis of EGFP expression in MC38 tumors revealed that intratumorally inoculated shIL-10 LVs transduced tumor-infiltrating myeloid cells with the highest efficiency and, led to a decreased IL-10 level in the tumor microenvironment. However, the effect was accompanied by increased influx of PMN-MDSCs into tumors observed both on the 6th and on the 10th day after shIL-10 LV injections. Nevertheless, it was noted that suppressor activity of myeloid cells isolated from tumors was dependent on the efficiency of
\end{abstract}

Correspondence to: Dr Natalia Anger-Góra or Dr Joanna Rossowska, Hirszfeld Institute of Immunology and Experimental Therapy, Polish Academy of Sciences, Weigla 12, 53-114 Wrocław, Poland

E-mail: natalia.anger@hirszfeld.pl

E-mail: joanna.rossowska@hirszfeld.pl

Key words: myeloid-derived suppressor cells, interleukin 10, tumor microenvironment, lentiviral vectors, cyclophosphamide, MC38 tumor-derived PMN-MDSC transduction with shIL-10 LVs. The increased percentage of transduced PMN-MDSCs on the 10th day was associated with diminished immunosuppressive activity of tumor-derived myeloid cells and an elevated ratio of cytotoxic T lymphocytes to M-MDSCs. The obtained data indicated that treatment with shIL-10 LVs may result in modulation of the immunosuppressive activity of MC38 colon carcinoma-derived MDSCs.

\section{Introduction}

Immunotherapy used in treatment of neoplastic diseases aims to boost the antitumor response, where antigen-specific T lymphocytes play an important role. However, the presence of immunosuppressive cells, such as myeloid-derived suppressor cells (MDSCs), tumor-associated macrophages (TAMs), regulatory $\mathrm{T}$ lymphocytes (Tregs) and soluble factors [e.g. interleukin-10 (IL-10), transforming growth factor- $\beta$ (TGF- $\beta$ ), vascular endothelial growth factor (VEGF), indoleamine 2,3-dioxygenase (IDO), prostaglandin E2 (PGE2)] in the tumor microenvironment (TME), contributes to the reduction of the activity of the effector T cells, thus diminishing the effectiveness of the applied treatment $(1,2)$. Among the most potent suppressors of antitumor immunity are myeloid-derived suppressor cells, a heterogenic population that consists of monocytic (M-MDSCs) and polymorphonuclear (PMN-MDSCs) cells, whose differentiation from myeloid precursors is altered by the tumor-derived factors $(3,4)$. During carcinogenesis, the accumulation of these cells can be observed in tumor sites and their frequency is often considered as a biomarker for predicting the response to the applied immunotherapy and prognosis of the clinical outcome in patients (5-7). MDSCs suppress the specific immune response by acting on immunocompetent cells and promoting escape of the tumor from immune surveillance, which leads to the progression of the disease. Depletion of amino acids necessary for T-cell activation, production of reactive oxygen and nitrogen species, and expression of proteins of a suppressive nature, such as IL-10, 
TGF- $\beta$ and programmed death-ligand 1 (PD-L1), by MDSCs lead to inhibition of the activity of antitumor immune cells, as well as induction of Tregs with suppressor activity (8).

Although recent reports indicate that IL-10 may take part in induction of MDSCs $(9,10)$, the role of TME-derived IL-10 in differentiation of MDSCs and activation of their suppression mechanisms is not fully understood. Based on the literature, it is known that IL-10 affects the activity of other myeloid cells in the TME. It hinders the maturation of dendritic cells (DCs) and reduces the expression of costimulatory molecules, limiting their ability to induce a specific antitumor response $(11,12)$. Moreover, it promotes polarization of macrophages towards M2-type cells, which in consequence produce elevated amounts of IL-10 that inhibits the secretion of IL-12 in autocrine fashion (13). In addition, IL-10 impairs the antitumor activity of $\mathrm{CD}^{+}$ $\mathrm{T}$ cells by reducing the secretion of Th1-type cytokines, as well as by inducing the differentiation of Tregs $(14,15)$. Since these processes invariably lead to tumor progression, IL-10 is regarded as a potential target for antitumor therapies (16). In our previous research, local elimination of IL-10 was applied in antitumor immunotherapy. Trials with cyclophosphamide (CY), dendritic cell-based vaccines and lentiviral vectors (LVs) locally silencing the expression of this cytokine revealed high efficiency of this kind of treatment in MC38 murine colon carcinoma growth inhibition and showed that the therapeutic effect was accompanied by a reduction of the percentage of MDSCs in tumors (17). However, it was also observed that a few days after application, intratumorally injected LVs carrying shRNA against IL-10 (shIL-10 LVs) induced an increased influx of PMN-MDSCs into tumors (17). Considering our previous observations, this study aimed to determine the mechanisms of acting of lentiviral vectors encoding shRNA against IL-10 with particular emphasis on their influence on the activity of tumor-derived MDSCs. Third-generation lentiviral vectors encoding shRNA were used to silence the expression of IL-10 in ex vivo-generated MDSCs, as well as to reduce the production of IL-10 in the TME of MC38 colon carcinoma. Lentiviral vectors were injected intratumorally according to two different schedules, which made it possible to demonstrate the kinetics of immune response induction after application of shIL-10 LVs, as well as to establish whether shIL-10 LVs were able to efficiently reduce the IL-10 secretion after development of antiviral immune response. Since cyclophosphamide was reported as an IL-10 reducing agent and due to its potential to increase the antitumor activity of some immunotherapeutic strategies (18), the intratumoral activity of shIL-10 LVs was also evaluated when they were applied in the combined treatment with a low dose of CY. The gathered data indicate that transduction with shIL-10 LVs results in altered immunosuppressive activity of ex vivo-generated and tumor-derived MDSCs. Reduction of immunosuppressive activity of myeloid cells infiltrating tumors depends on the direct influence of the vectors on PMN-MDSCs, which were observed to lose their suppressor activity following transduction with shIL-10 LVs in in vitro conditions. Nevertheless, this effect appears to be dependent both on specific silencing of IL-10 expression and changes mediated by lentiviral transduction itself.

\section{Materials and methods}

Mice. Female C57BL/6 mice were obtained from the Center for Experimental Medicine of the Medical University of Bialystok, Poland. All experiments were performed in accordance with Directive 2010/63/EU of the European Parliament and of the Council on the protection of animals used for scientific purposes and were approved by the 1st Local Ethic Committee for Experiments with the Use of Laboratory Animals, Wroclaw, Poland (authorization number 11/2015, $33 / 2018$ ). After the experiments, mice were sacrificed by cervical dislocation.

Cell culture. The in vivo growing MC38 murine colon carcinoma from the Tumor Bank of the TNA Radiobiology Institute (Rijswijk, The Netherlands) was adapted to in vitro conditions as described by Pajtasz-Piasecka et al (19). MC38/0 cells were maintained in RPMI-1640 (Gibco) supplemented with $100 \mathrm{U} / \mathrm{ml}$ penicillin, $10 \mathrm{mg} / \mathrm{ml}$ streptomycin, $1 \mathrm{mM}$ sodium pyruvate, $0.05 \mathrm{mM}$ 2-mercaptoethanol and $5 \%$ fetal bovine serum (FBS; all reagents from Sigma-Aldrich). Lenti-X 293T cell line (Clontech) was maintained in high-glucose Dulbecco's Modified Eagle Medium (Gibco) supplemented with $100 \mathrm{U} / \mathrm{ml}$ penicillin, $100 \mathrm{mg} / \mathrm{ml}$ streptomycin, $1 \mathrm{mM}$ sodium pyruvate and $10 \%$ of FBS. MDSCs were generated from bone marrow of healthy (wild-type) C57BL/6 mice isolated according to the procedure described by Rossowska et al (20). The cells were cultured in a conditioned medium consisting of $25 \%$ RPMI 1640 and 75\% supernatant from the above MC38/0 cells $\left(10 \times 10^{6} / 10 \mathrm{ml}\right)$ cultured in hypoxia conditions $\left(1 \% \mathrm{O}_{2}\right)$ for $48 \mathrm{~h}$, supplemented with $10 \%$ FBS and $80 \mathrm{ng} / \mathrm{ml}$ recombinant murine granulocyte-macrophage colony-stimulating factor (rm GM-CSF) (ImmunoTools). The medium was replaced every two days of culture. On the 6th day, MDSCs were transduced with lentiviral vectors in the presence of polybrene $(10 \mu \mathrm{g} / \mathrm{ml}$, Sigma-Aldrich).

Production of lentiviral vectors. Lentiviral vectors (LVs) were produced using a third-generation lentiviral system, which consisted of pMDLg/pRRE, pRSV-Rev, pMD2.G [the plasmids were a gift from Didier Trono (Addgene plasmid \#12251, 12253, 12259)] and pGLV-H1-GFP + Puro expression plasmids (EzBiolab). The expression plasmids encoded three different shRNA sequences against IL-10 (shIL-10) or a non-targeting sequence of shRNA against human GAPDH (shN), which was used as a negative control (Fig. 1). Lentiviral vectors were produced and concentrated according to the procedure described in our previous article (21). Briefly, Lenti-X cells were co-transfected with plasmids and cultured for $48 \mathrm{~h}$. Supernatant containing lentiviral vectors was collected and concentrated by precipitation using PEG 6000 (Sigma-Aldrich). The titer of the lentiviral vectors was determined by the serial dilution method using MC38/0 cells.

Determining IL-10 silencing efficiency in transduced MDSCs. MDSCs were transduced with LVs encoding shRNA silencing the expression of IL-10. For reference, non-transduced cells, or cells transduced with control LVs encoding non-targeting shRNA against human GAPDH, were used. After 3 days, the efficacy of transduction was determined by flow cytometry as 
a percentage of enhanced green fluorescent protein-positive $\left(\mathrm{EGFP}^{+}\right)$cells. The efficiency of IL-10 silencing was evaluated at mRNA and protein levels using real-time PCR and ELISA, respectively. Total RNA was isolated using a NucleoSpin RNA kit (Macherey-Nagel) and reverse-transcribed with a RevertAid First Strand cDNA Synthesis Kit (Thermo Scientific). Real-time PCR was performed using TaqMan Universal PCR Master Mix and TaqMan Gene Expression Assay primers for IL-10 (Applied Biosystems) in reference to the HPRT gene. Concentration of IL-10 in supernatants from the MDSC 24-h culture established on the $3 \mathrm{rd}$ day after transduction $\left(0.25 \times 10^{6}\right.$ cells $\left./ 0.5 \mathrm{ml}\right)$ was measured by ELISA (BD Biosciences).

Estimation of differentiation level of transduced MDSCs. Three days after transduction, the phenotype characterization of MDSCs was performed by flow cytometry. The cells were labeled with a cocktail of monoclonal antibodies conjugated with fluorochromes: Anti-CD11b (M1/70) PerCP-Cy5.5, anti-CD11c (N418) Brilliant Violet (BV) 650, anti-Ly6C (HK1.4) BV 510, anti-Ly6G (1A8) BV 605, anti-MHC II (M5/114.15.2) APC-Cy7, anti-CD86 (GL-1) PE-Cy7, anti-F4/80 (BM8) Alexa Fluor 700, anti-PD-L1 (10F.9G2) APC (all from BioLegend). Analysis of the cell phenotype was performed using LSRFortessa flow cytometer with FACS Diva software (BD Biosciences). In order to exclude dead cells, DAPI dye (Invitrogen) was added prior to the analysis.

Determination of suppressive activity of transduced MDSCs. Spleen cells obtained from healthy (wild-type) C57BL/6 mice were labeled with CellTrace CFSE (carboxyfluorescein succinimidyl ester) dye $\left(0.5 \mu \mathrm{M}, 15 \mathrm{~min}, 37^{\circ} \mathrm{C}\right.$; Invitrogen $)$ and co-cultured with transduced M-MDSCs or PMN-MDSCs sorted with FACS Aria (BD Biosciences). Cells were cultured in a ratio of $1: 1\left(1 \times 10^{5}\right.$ splenocytes and $1 \times 10^{5}$ M-MDSCs or $1 \times 10^{4}$ splenocytes and $1 \times 10^{4}$ PMN-MDSCs) or $2: 1\left(2 \times 10^{5}\right.$ splenocytes and $1 \times 10^{5}$ M-MDSCs or $2 \times 10^{4}$ splenocytes and $1 \times 10^{4}$ PMN-MDSCs) in the presence of concanavalin A ( $2 \mu \mathrm{g} / \mathrm{ml}$; Sigma-Aldrich) and rh IL-2 (200 U/ml,ImmunoTools). After 3 days, the cells were labeled with monoclonal antibodies conjugated with fluorochromes: Anti-CD11b (M1/70) PerCP-Cy5.5, anti-CD4 (RM4-5) APC, anti-CD8 (53-6.7) $\mathrm{PE}-\mathrm{Cy}$ (all from BioLegend). CFSE mean fluorescence intensity (MFI) in $\mathrm{CD}^{+}$and $\mathrm{CD}^{+}$cells was measured using the LSRFortessa (BD Biosciences). Concentrations of interferon- $\gamma$ (IFN- $\gamma)$ and IL-10 in supernatants from the above co-culture were determined using ELISA kits (eBioscience, Invitrogen and BD Biosciences, respectively).

Intratumoral application of lentiviral vectors. Eight to ten-week-old female C57BL/6 mice were subcutaneously inoculated in the right flank with MC38/0 cells $\left(1.1 \times 10^{6}\right.$ cells $\left./ 0.2 \mathrm{ml} / \mathrm{mouse}\right)$. After the tumor volume had reached $\sim 50 \mathrm{~mm}^{3}$, mice were randomized and treated with cyclophosphamide (CY) and/or LVs encoding shRNA against IL-10 (shIL-10 LVs) or with LVs encoding non-targeting shRNA against human GAPDH (shN LVs). Lentiviral vectors were applied intratumorally (i.t.; $2 \times 10^{6} \mathrm{TU} / 50 \mu \mathrm{l} /$ mouse) three times, accordingly to the schemes presented in the Results section. The intratumoral injections were performed with extreme caution and any mechanical disruption of the tumor tissue were not observed. Cyclophosphamide (Baxter) was applied i.p. (150 mg/kg body weight) two days prior to the first LV injection. On the 6th (7-10 mice per group) or 10th (7-10 mice per group) day of the treatment (LVs) and on the 8th (3 mice per group) and 12th (9-10 mice per group) day of the treatment $(\mathrm{CY}+\mathrm{LVs})$, mice were sacrificed, and tumors and tumor-draining lymph nodes (tLNs) were dissected, homogenized and used for further analyses.

Determination of IL-10 silencing efficiency in tumors. Concentration of IL-10 in supernatants from 24-h culture of cells isolated from the tumor tissue $(10 \mathrm{mg} / \mathrm{ml})$ was estimated using ELISA (BD Biosciences).

Analysis of myeloid cell populations in tumors of LV-treated mice. Tumor cells were isolated from mice were labeled with a cocktail of monoclonal antibodies conjugated with fluorochromes: Anti-CD45 (30-F11) V500, anti-CD3 (145-2C11) PE-CF594, anti-CD19 (1D3) PE-CF594, anti-CD49b (DX5) PE-CF594 (all from BD Biosciences), anti-CD11b (M1/70) PerCP-Cy5.5, anti-CD11c (N418) BV 650, anti-F4/80 (BM8) Alexa Fluor 700, anti-Ly6C (HK1.4) PE, anti-Ly6G (1A8) BV 605 (all from BioLegend). In order to exclude dead cells, DAPI dye was added prior to the analysis. Analysis of percentage of tumor-infiltrating myeloid cells as well as EGFP expression was performed using the LSRFortessa (BD Biosciences).

Analysis of lymphoid cell populations in tumors and tumor-draining LNs of LV-treated mice. Tumor and lymph node cells isolated from mice were stained with LIVE/DEAD Fixable Violet Dead Staining Kit (Invitrogen) and labeled with a cocktail of monoclonal antibodies conjugated with fluorochromes: Anti-CD45 (30-F11) BV 605, anti-CD3 (17A2) BV 650, anti-CD4 (RM4-5) FITC, anti-CD8 (53-6.7) APC/Fire750, anti-CD25 (PC61) PE, anti-CD44 (IM7) PE-Cy7, anti-CD62L (MEL-14) PerCP-Cy5.5 (all from BioLegend), and anti-CD49b (DX5) PE-CF594 (BD Biosciences) for lymphoid cell analysis in tumors and anti-CD4 (RM4-5) PerCP-Cy5.5, anti-CD8 (53-6.7) PE-Cy, anti-CD44 (IM7) FITC, and anti-CD62L (MEL-14) BV 605 (all from BioLegend) for lymphocyte analysis in tLNs. In the following step, the cells were fixed using eBioscience Foxp3/Transcription Factor Staining Buffer Set (Invitrogen). Subsequently, cells from tLNs were labeled with anti-Ki67 (16A8) APC (BioLegend). Analyses of lymphoid cells in tumors and tLNs were performed using LSRFortessa (BD Biosciences).

Determination of suppressive activity of tumor-infiltrating myeloid cells. Tumor cells isolated from mice were labeled with anti-CD11b monoclonal antibody (M1/70) conjugated with magnetic nanoparticles (BD IMag). Myeloid (CD11b $\left.{ }^{+}\right)$ cells were magnetically separated and co-cultured with CFSE-labeled splenocytes from healthy mice in a ratio of 1:1 $\left(5 \times 10^{4}\right.$ tumor-infiltrating CD11b ${ }^{+}$cells and $5 \times 10^{4}$ splenocytes). Co-culture as well as the analysis was performed according to the procedure described above. CFSE mean fluorescence intensity $(\mathrm{MFI})$ in $\mathrm{CD}^{+}$and $\mathrm{CD}^{+}$cells was measured using the LSRFortessa (BD Biosciences). Concentrations of IFN- $\gamma$ and IL-10 in supernatants from the co-culture were determined using ELISA kits (eBioscience, Invitrogen and BD Biosciences, respectively). 
Statistical analysis. All the data were analyzed using GraphPad Prism 8 software. Normality was verified using the Shapiro-Wilk test. Statistical significance of normally distributed data was calculated using Welch's ANOVA followed by post hoc Dunnett's T3 multiple comparison test. For the analysis of non-normally distributed data, the non-parametric Kruskal-Wallis test followed by post hoc Dunn's multiple comparison test or the Mann-Whitney U test was used. $\mathrm{P}<0.05$ was considered to indicate a statistically significant difference.

\section{Results}

IL-10 silencing in in vitro-cultured MDSCs induced changes in their suppressive activity. In the first step of the study, the effectiveness of three different nucleotide sequences of shRNA in silencing of murine IL-10 was determined. The research was conducted using MDSCs, which were ex vivo-generated during six-day culture of bone marrow cells in the presence of GM-CSF and the supernatant collected from MC38 colon carcinoma culture maintained in hypoxia conditions. Phenotype characterization of the obtained cells was performed using the flow cytometry method according to the scheme presented in Fig. 1A. On the 6th day of differentiating cell culture, M-MDSCs $\left(\mathrm{CD} 11 b^{+} \mathrm{CD} 11 \mathrm{c}^{\text {neg }} \mathrm{Ly} 6 \mathrm{C}^{+} \mathrm{Ly}_{6 \mathrm{G}}{ }^{\mathrm{neg}}\right)$ constituted the vast majority in the cell suspension (over 60\%), whereas PMN-MDSCs (CD11b+CD11c ${ }^{\text {neg }}$ Ly6C $\left.^{\text {int }} \mathrm{Ly}^{+} \mathrm{G}^{+}\right)$ accounted for approx. 20\% of the cells (Fig. 1A). Apart from MDSCs, CD11c cells corresponding to immature dendritic cells were also detected (approximately $10 \%$ of all cells). As expected, the obtained MDSCs were characterized by lower expression of molecules associated with the maturation stage of myeloid cells such as MHC class II, CD86, F4/80 or PD-L1 compared to $\mathrm{CD} 11 \mathrm{c}^{+}$cells. Such phenotype characteristics indicate typical features of MDSCs (Fig. 1A).

The obtained cell suspension was transduced using lentiviral vectors encoding EGFP as a reporter gene and shRNAs against murine IL-10 (Fig. 1B and C). Transduced cells were characterized by high expression of EGFP and significantly reduced expression of IL-10 (Fig. 1D-F). Real-time PCR and ELISA confirmed that the shIL-10-3 sequence was the most effective in IL-10 silencing, and hence this sequence was chosen for further experiments where it was referred to as shIL-10.

As a result of transduction with shN or shIL-10 LVs, decreased percentages of M-MDSCs and PMN-MDSCs in the cell suspension were detected. However, the efficiency of transduction in both subpopulations of MDSCs, presented here as a percentage of $\mathrm{EGFP}^{+}$cells, was high (Fig. 2A). Although we observed a statistically significant shift in the expression of selected molecules on the surface of modified M-MDSCs and PMN-MDSCs in relation to non-transduced cells, these changes were not dependent on the IL-10 silencing but were related to lentiviral transduction itself (Figs. 2B and S1). Moreover, compared to the high expression of these molecules on CD11c ${ }^{+}$cells (Fig. S2A and B), it may be concluded that neither M-nor PMN-MDSCs undergo substantial phenotypical changes under the influence of lentiviral transduction and they retain the status of immature cells.

The influence of IL-10 silencing on the suppressor activity of in vitro-cultured M-MDSCs and PMN-MDSCs was determined using a CFSE-based proliferation assay (Figs. 3, 4 and S3). Since these two subpopulations employ different mechanisms to suppress $\mathrm{T}$ cell activity, we decided to evaluate their function separately.

The obtained data showed that the proliferation of spleen-derived $\mathrm{CD}^{+}$and $\mathrm{CD}^{+} \mathrm{T}$ lymphocytes was the lowest when cells were co-cultured with M-MDSC/shIL-10 (Figs. 3A and B, and S4A). Statistically significant differences were detected between non-transduced M-MDSCs and M-MDSC/shIL-10 groups (Fig. 3A). It is worth noting that transduction with shN LVs also resulted in significantly increased suppressive activity of MDSCs. The highly suppressive activity of M-MDSC/shIL-10 was also reflected in the production of cytokines by $\mathrm{T}$ lymphocytes. Namely, splenocytes co-cultured with M-MDSC/shIL-10 secreted decreased amounts of IFN- $\gamma$ in comparison to those co-cultured with M-MDSCs or M-MDSC/shN (Fig. 3C and D). On the other hand, the production of IL-10 in this group was comparable to that obtained from the positive control of splenocytes cultured without M-MDSCs (splc ctrl) and considerably lower than in M-MDSCs or M-MDSC/shN groups (Fig. 3C and D).

The opposite effect was noted when splenocytes were cultured in the presence of PMN-MDSC/shIL-10. The obtained results indicate that PMN-MDSC/shIL-10 acts more as an inducer of $\mathrm{CD}^{+}$and $\mathrm{CD}^{+} \mathrm{T}$ lymphocyte proliferation than as a suppressor (Fig. 4A and B). Although the differences between groups were not statistically significant, it should be noted that splenocytes co-cultured in the presence of PMN-MDSC/shIL-10 (in both a 1:1 and a 2:1 ratio) proliferated even more intensively compared to control splenocytes, whereas proliferation in the PMN-MDSC group was lower than in the splc ctrl group or more or less at the level of the positive control in the case of the PMN-MDSC/shN group (Fig. 4A and B). Moreover, a statistically significant difference of $\mathrm{CD}^{+} \mathrm{T}$ lymphocyte proliferation when cultured in the presence of PMN-MDSCs and PMN-MDSC/shIL-10 in a ratio of 4:1 was detected (Fig. S4B). Additionally, PMN-MDSC/shIL-10 induced higher production of IFN- $\gamma$ compared to the PMN/MDSC group, and the difference was statistically significant when splenocytes were co-cultured with PMN-MDSCs at a ratio of 2:1, which was not observed in the co-culture of splc and PMN-MDSC/shN (Fig. 4D). Production of IL-10 in the PMN-MDSC/shIL-10 group was comparable to the splc ctrl group and considerably lower than in the PMN-MDSC group (at 1:1 ratio) and PMN-MDSC/shN group (Fig. 4C and D).

The obtained data indicate that introduction of shRNA against IL-10 into M-MDSCs and PMN-MDSCs did not induce the maturation of the cells towards fully functional myeloid cells such as macrophages, dendritic cells, or granulocytes. However, transduction of particular subpopulations of MDSCs with shIL-10 LVs resulted in changes of their suppressor activity. Namely, it was observed that M-MDSCs transduced with shIL-10 LVs showed increased suppressor activity in comparison to untransduced cells. The opposite effect was observed for the PMN-MDSC subpopulation-their transduction with shIL-10 LVs resulted in decreased ability to suppress $\mathrm{T}$ cell activity. Since MDSC/shN were also characterized by lowered expression of IL-10 in comparison to untransduced cells, the suppressor activity of MDSC/shIL-10 did not differ significantly from MDSC/shN. However, it was observed that the effect of transduction was correlated with the efficiency of reduction of IL-10 expression. 

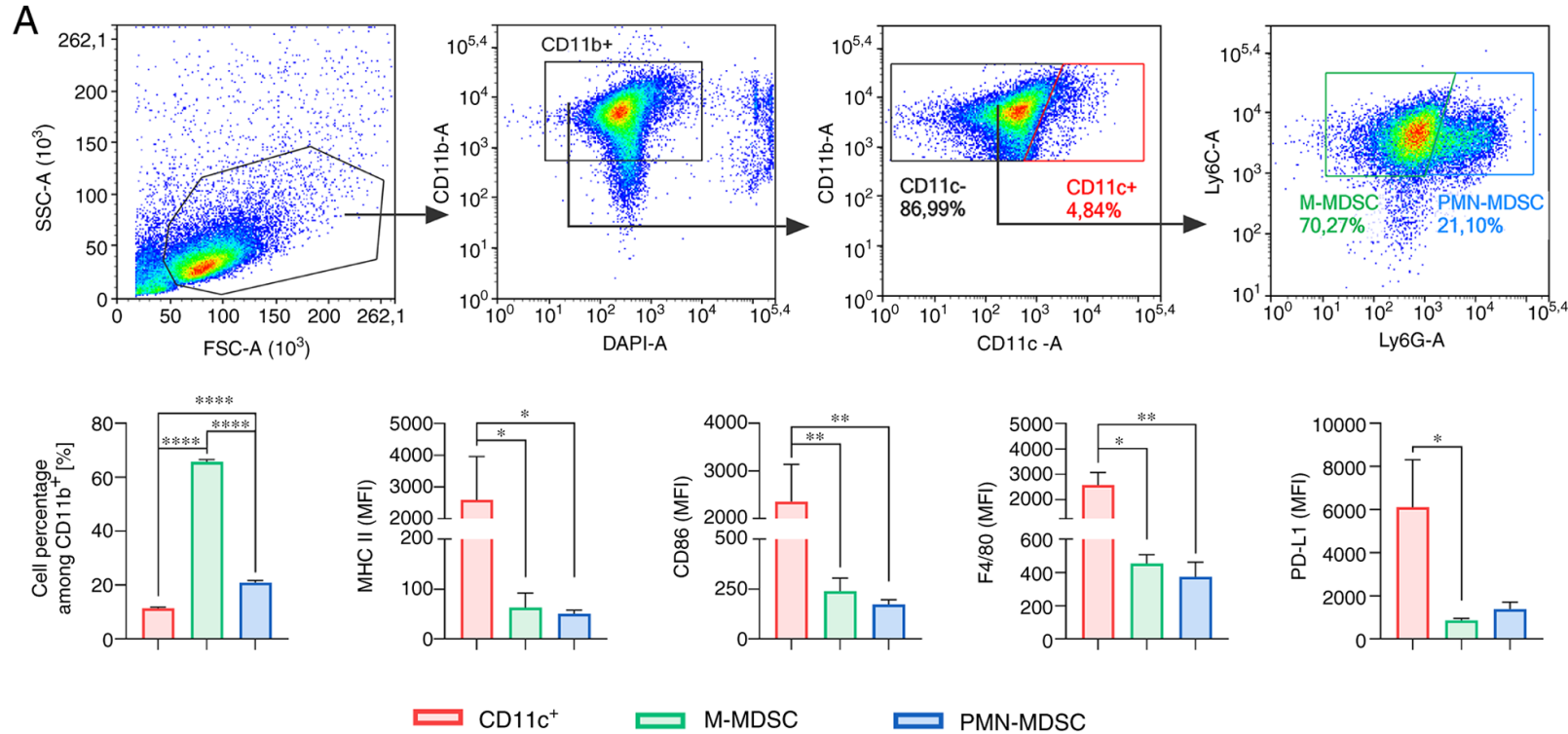

\section{P PMN-MDSC}

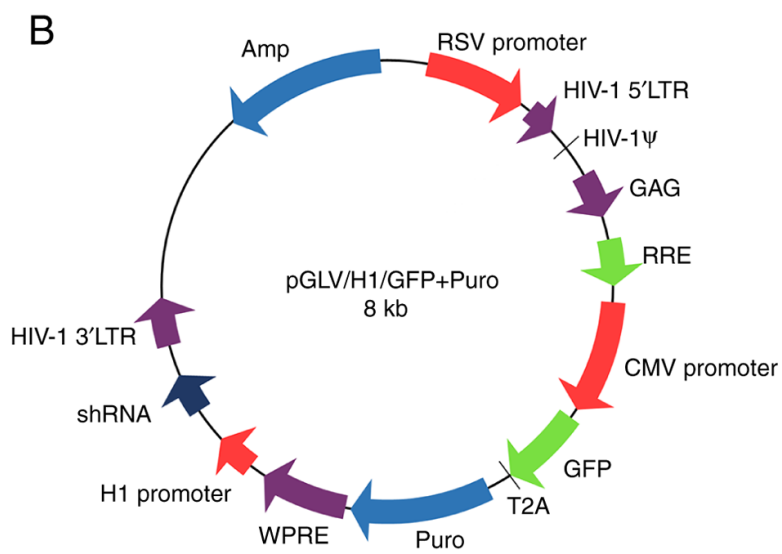

E
C

\begin{tabular}{lc} 
Vector & \multicolumn{1}{c}{ Targeted sequence } \\
\hline shN & 5'-TATGACAACAGCCTCAAG-3' \\
shIL-10-1 & 5'-GGTGAAGACTTTCTTTCAAAC-3' \\
shIL-10-2 & 5'-GCCAAGCCTTATCGGAAATGA-3' \\
shIL-10-3 & 5'-GGGATCTTAGCTAACGGAAAC-3'
\end{tabular}

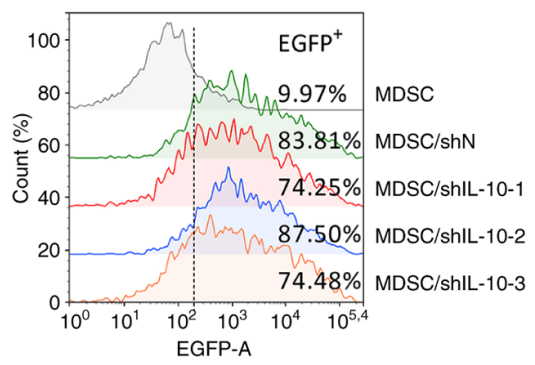

D

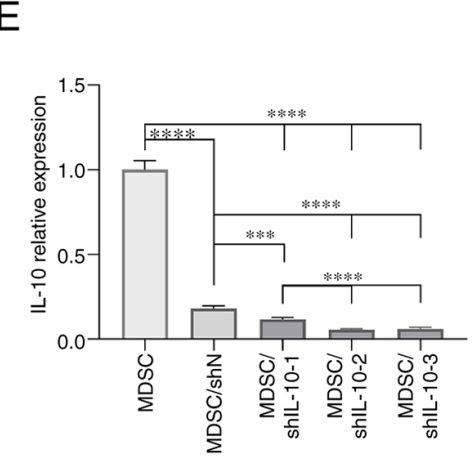

F

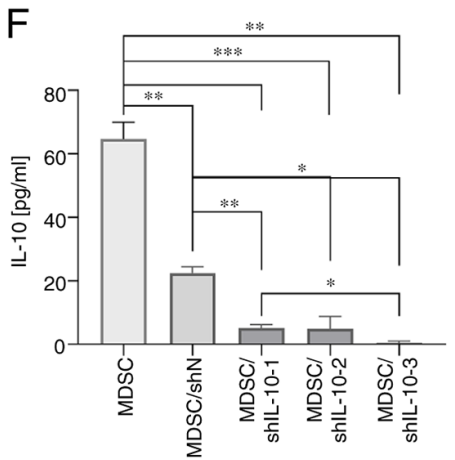

Figure 1. Effectiveness of IL-10 silencing in MDSCs after transduction with LVs encoding shRNA for IL-10. (A) The scheme of cytometric analysis and phenotype characteristics of MDSCs obtained from a 6-day culture of murine bone marrow cells maintained in conditioned medium and in the presence of rm GM-CSF. The results are given as the mean \pm SD calculated for two independent experiments measured in triplicate. (B) Scheme of a lentiviral vector encoding shRNA. (C) Targeted nucleotide sequences of selected shRNA specific for murine IL-10 (shIL-10) and non-targeting control sequence $(\mathrm{shN})$. (D) EGFP expression in MDSCs on the 3rd day after transduction with LVs. (E) Relative expression of IL-10 mRNA in transduced cells examined on the 3rd day after transduction with LVs using real-time PCR. The results are given as the mean \pm SD calculated for two independent experiments measured in triplicate (F) Concentration of IL-10 in supernatants collected after 24 h culture of shIL-10 or shN transduced MDSCs, established on the 3rd day after transduction, measured using ELISA. The results are given as the mean \pm SD calculated for three independent experiments measured in duplicates. Statistical significance was calculated using Welch's ANOVA followed by post hoc Dunnett's T3 multiple comparison test. Differences with a P<0.05 were regarded as significant $\left({ }^{*} \mathrm{P}<0.05,{ }^{* *} \mathrm{P}<0.01,{ }^{* * *} \mathrm{P}<0.001,{ }^{* * * *} \mathrm{P}<0.0001\right)$. MFI, mean fluorescence intensity; M-MDSC, monocytic myeloid-derived suppressor cells; PMN-MDSC, polymorphonuclear myeloid-derived suppressor cells; EGFP, enhanced green fluorescent protein.

Intratumoral injections of shIL-10 LVs reduced IL-10 production in TME and influenced the proportions and activity of myeloid cells infiltrating tumor. In order to determine the influence of lentiviral vectors carrying shRNA against IL-10 on the activity of immune cells in tumor tissue, shIL-10 LVs and control shN LVs were administered to MC38 tumor-bearing 
A
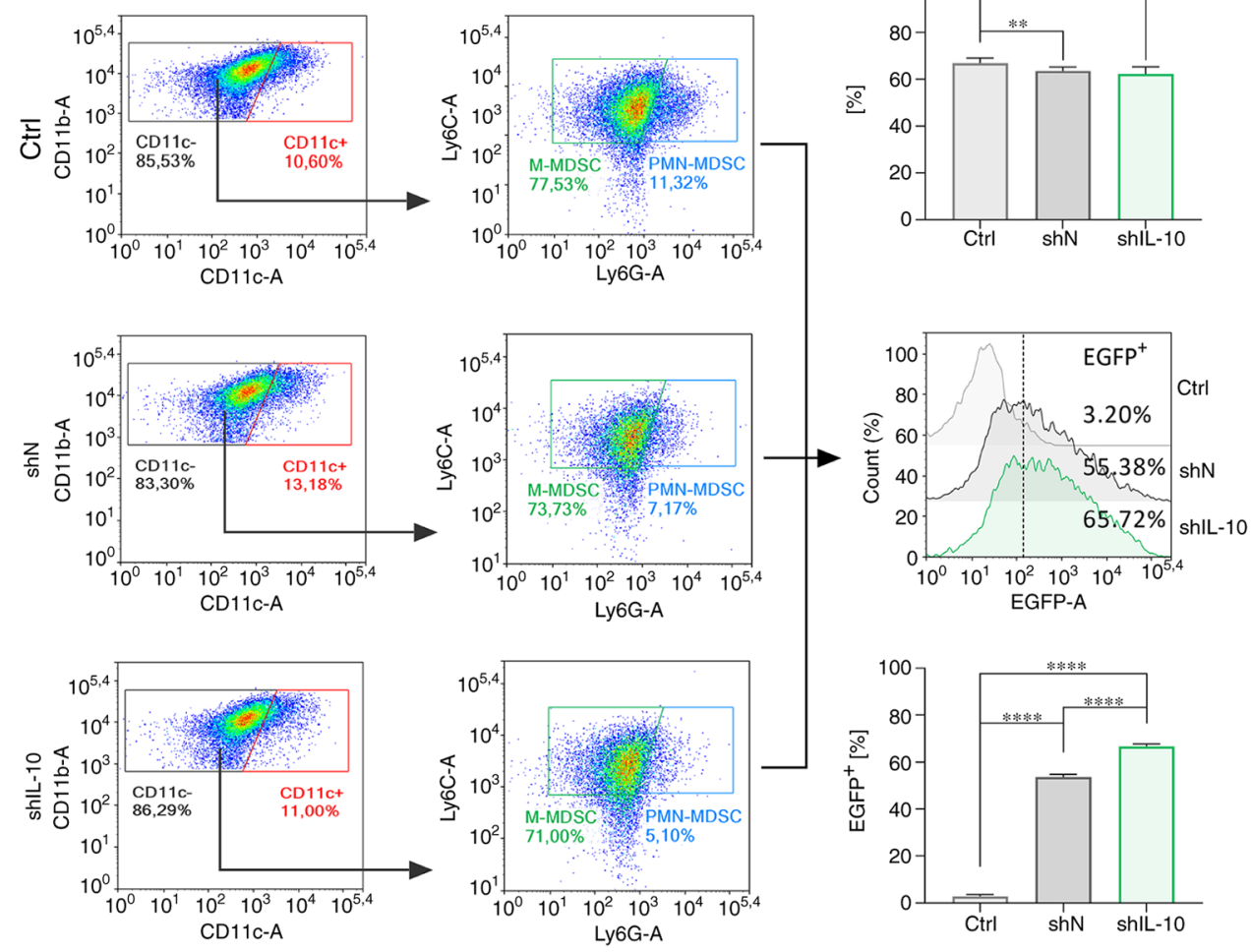
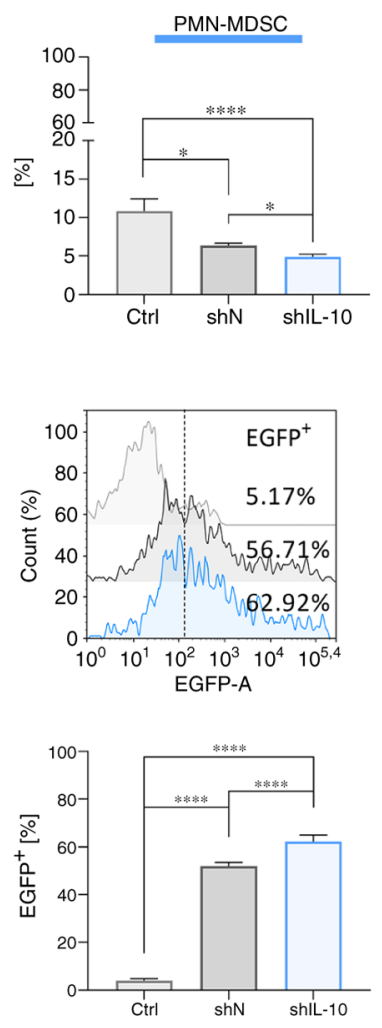

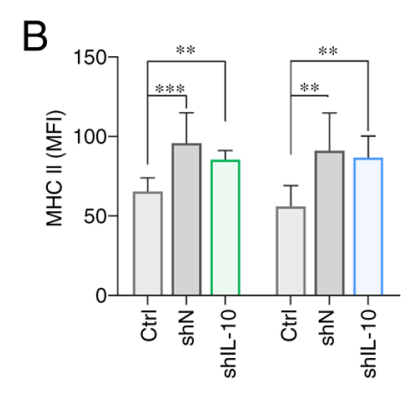

$\overline{M-M D S C} \quad \overline{\text { PMN-MDSC }}$

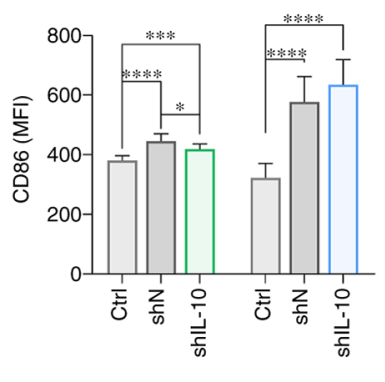

M-MDSC PMN-MDSC

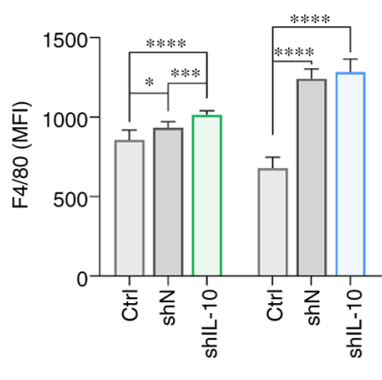

M-MDSC PMN-MDSC

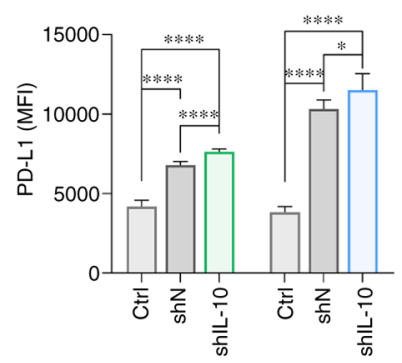

M-MDSC PMN-MDSC

Figure 2. Characteristics of M-MDSCs and PMN-MDSCs with the silenced expression of IL-10. (A) Representative flow cytometric data and bar plots

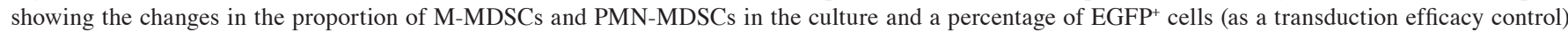
among identified subpopulations of MDSCs on the 3rd day after IL-10 silencing. (B) Phenotype characteristics of M-MDSCs and PMN-MDSCs on the 3rd day after IL-10 silencing. The results are given as the mean \pm SD calculated for three independent experiments measured in triplicates. Statistical significance was calculated using Welch's ANOVA followed by post hoc Dunnett's T3 multiple comparison test. Differences with a $\mathrm{P}<0.05$ were regarded as significant $\left({ }^{*} \mathrm{P}<0.05,{ }^{* *} \mathrm{P}<0.01,{ }^{* * *} \mathrm{P}<0.001,{ }^{* * * *} \mathrm{P}<0.0001\right)$. M-MDSC, monocytic myeloid-derived suppressor cells; PMN-MDSC, polymorphonuclear myeloid-derived suppressor cells; EGFP, enhanced green fluorescent protein; ctrl, non-transduced cells; shN, cells transduced with LVs encoding shN sequence; shIL-10, cells transduced with LVs encoding shIL-10 sequence; MFI, mean fluorescence intensity.

mice. Triple, intratumoral injections of the vectors were conducted according to the schemes presented in Fig. 5A and B. Tumors and tumor-draining lymph nodes were harvested on the 6th or 10th day starting from the first LV injection. It was observed that the concentration of IL-10 produced by cells isolated from tumors dissected on both the 6th and 10th day was substantially lower in the group that received shIL-10 LVs compared to the untreated control and the shN LV group (Fig. 5C and D). Detailed analysis of EGFP expression in tumor cells (characterized here as CD45 ${ }^{\text {neg }}$ ), as well as lymphoid and myeloid cells infiltrating the TME, revealed that on the 6th day after $\mathrm{LV}$ treatment the $\mathrm{CD}_{11 \mathrm{~b}^{+}}$myeloid cells were the only population with enhanced expression of EGFP, whereas the 10th day myeloid cells in LV groups were characterized by nearly two-fold higher EGFP expression than in the control group (Figs. S5A and B, and 5E). In the prolonged scheme of treatment, enhanced expression of EGFP in tumor cells (Fig. S5A and B) was also observed. Further analyses, carried out on selected subpopulations of myeloid cells, demonstrated that on the 6th day the percentages of $\mathrm{EGFP}^{+}$cells were the highest in the M-MDSC and PMN-MDSC populations from the shIL-10 LV group and were at the level of 12.4 and $15.2 \%$ respectively (Fig. 5F). On the 10th day, a substantial increase in the percentage of PMN-MDSC/EGFP ${ }^{+}$was observed in both the shN LV group (up to $35.0 \%$ ) and the shIL-10 LV group (up to $30.6 \%$ ) compared to untreated control (Fig. 5G). The 
A
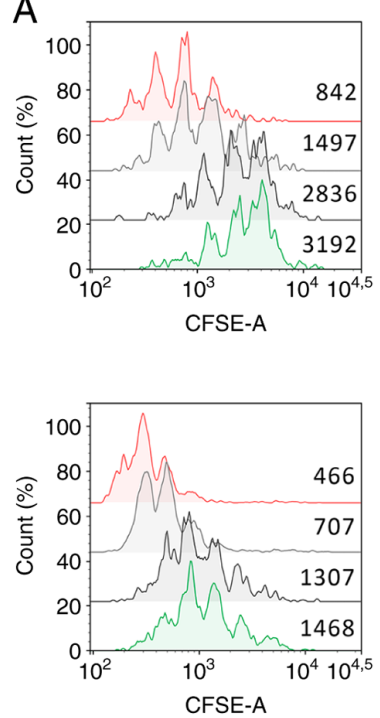

C

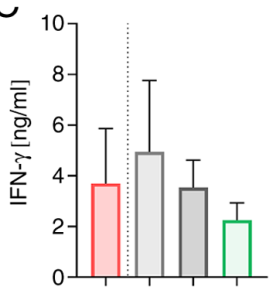

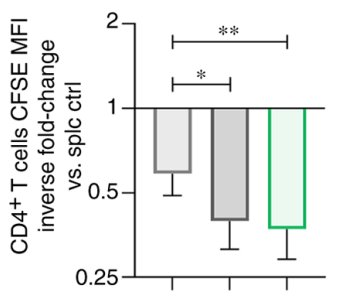
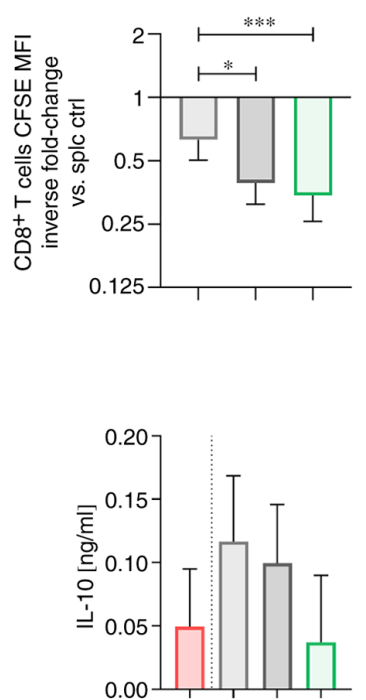

M-MDSC

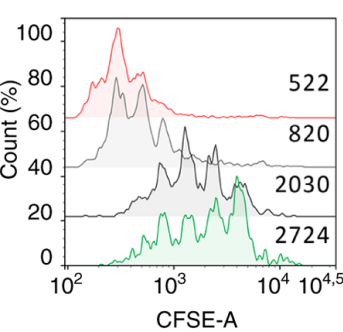

D

B
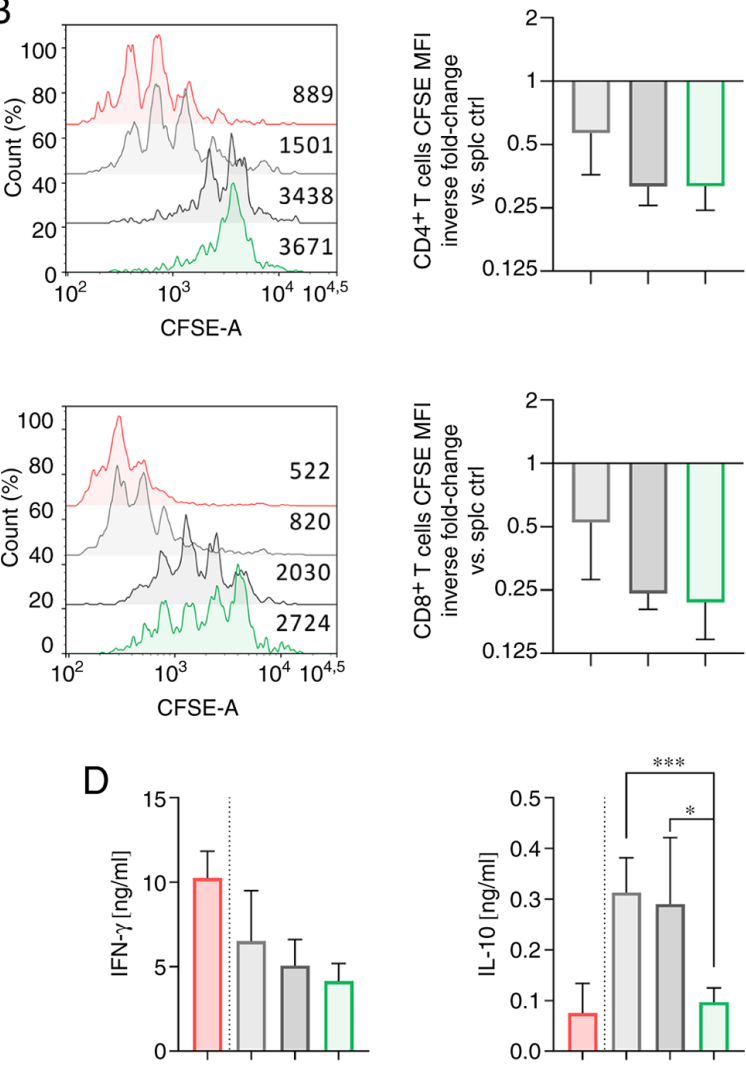

Splc ctrl

M-MDSC/shIL-10

Figure 3. Influence of IL-10 silencing on the suppressive activity of M-MDSCs. CFSE-labeled splenocytes obtained from healthy mice were cultured with M-MDSCs (isolated from the in vitro culture of MDSCs using the FACS Aria sorter) at the final ratio of (A and C) 1:1 or (B and D) 2:1. (A and B) Proliferation of $\mathrm{CD}^{+}$and $\mathrm{CD}^{+}$spleen-derived $\mathrm{T}$ lymphocytes was measured by CFSE dilution and fluorescence intensity on representative histograms. Splenocytes cultured with ConA and IL-2 (splc ctrl) were used as a positive control of proliferation. The results are presented as the mean inverse fold-change of CFSE MFI calculated in reference to the splc ctrl group \pm SD [100\% splc ctrl MFI value-3089 and 1005 (A, CD4+, exp. 1 and exp. 2 , respectively), 1679 and 475 (A, CD8 ${ }^{+}$, exp. 1 and exp. 2, respectively), 2882 and 1018 (B, CD4 ${ }^{+}$, exp. 1 and exp. 2, respectively), 1440 and 457 (B, CD8 ${ }^{+}$, exp.1 and exp. 2 , respectively)]. (C and D) Concentrations of IFN- $\gamma$ and IL-10 in supernatants collected after $72 \mathrm{~h}$ co-culture of M-MDSCs with splenocytes measured using ELISA. The results are expressed as the mean \pm SD. Data obtained for two independent experiments measured in triplicate. Statistical significance was calculated using Welch's ANOVA followed by post hoc Dunnett's T3 multiple comparison test. Differences with a $\mathrm{P}<0.05$ were regarded as significant $\left({ }^{*} \mathrm{P}<0.05,{ }^{* *} \mathrm{P}<0.01,{ }^{* * *} \mathrm{P}<0.001\right)$. M-MDSC, monocytic myeloid-derived suppressor cells; MFI, mean fluorescence intensity.

effectiveness of LV transduction in this subpopulation was considerably higher than in M-MDSCs (Fig. 5F and G). In the prolonged scheme of treatment, LVs demonstrated a noticeable increase in the effectiveness of DC modification. In both the shN and shIL-10 groups, the percentage of $\mathrm{DC}^{-\mathrm{EGFP}^{+}}$was high and varied within the range of $32-37 \%$ (Fig. 5G). It indicates that in the proposed scheme of treatment myeloid cells infiltrating MC38 tumors seem to be one of the main recipients of LV transduction in situ.

The analysis of changes in myeloid cell influx into MC38 tumors following the administrations of shIL-10 LVs was performed using the multiparameter flow cytometry method according to the scheme presented in Supplemental Fig. S6. The obtained data demonstrated a significant reduction of the percentage of $\mathrm{CD}_{11} \mathrm{~b}^{+}$in the aftermath of $\mathrm{LV}$ injections compared to the untreated control. The effect was noted on both the 6th and 10th day and there were no considerable differences between shN and shIL-10 LVs (Fig. 5H). A detailed analysis showed that LVs induced a significant reduction of TAM and DC populations in tumors compared to the untreated control group (Fig. 5I). In the case of DCs, the effect was the most intensive after injection of shIL-10 LVs and the percentage of the cells decreased over time. In tumors inoculated with shIL-10 LVs a lower percentage of M-MDSCs than in the untreated control and in the shN LV group was also detected, which was particularly evident on the 10th day after the first LV injection. Interestingly, shIL-10 LVs induced an increased influx of PMN-MDSCs into tumors. The highest percentage of these cells was noted on the 6th day and although it decreased over time, on the 10th day the proportions of these cells in the shIL-10 LV group were still significantly higher than in the control group (Fig. 5I).

The CFSE-based proliferation assay showed that myeloid cells isolated from shIL-10 LV-treated tumors dissected on the 6th day were characterized by considerably higher suppressor activity toward $\mathrm{CD}^{+} \mathrm{T}$ lymphocytes than those isolated from the untreated control or the shN LV group (Fig. 5J). However, it needs to be highlighted that the effect was temporary and on the 10th day after the first injection of shIL-10 LVs myeloid cells lost their suppressor activity in contrast to those obtained from the untreated group and the proliferation of $\mathrm{CD}^{+}$, as well as $\mathrm{CD}^{+} \mathrm{T}$ lymphocytes was even more intensive than 
A
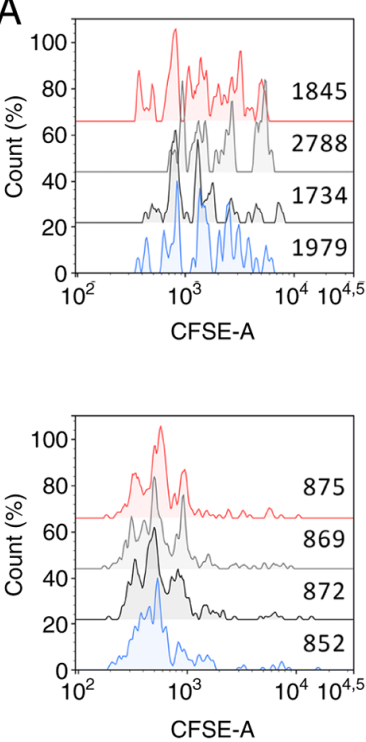

C

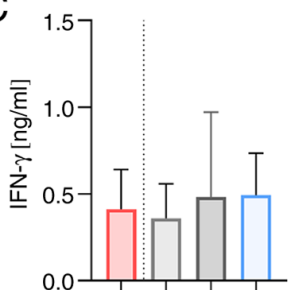

Splc ctrl
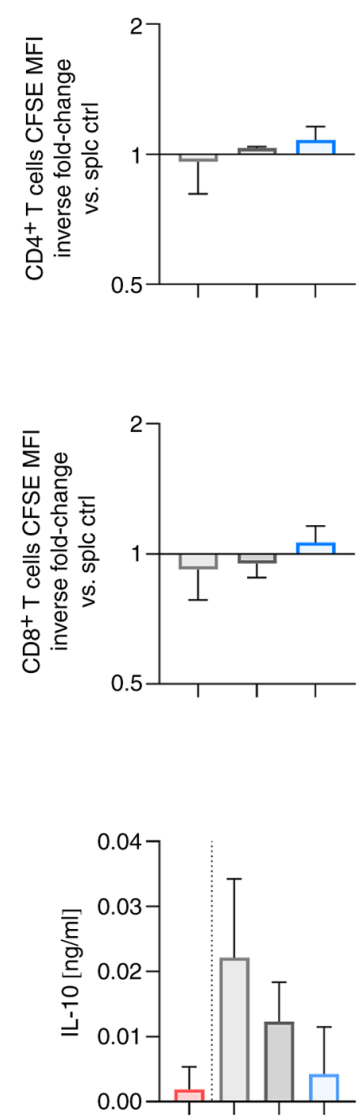

PMN-MDSC
B
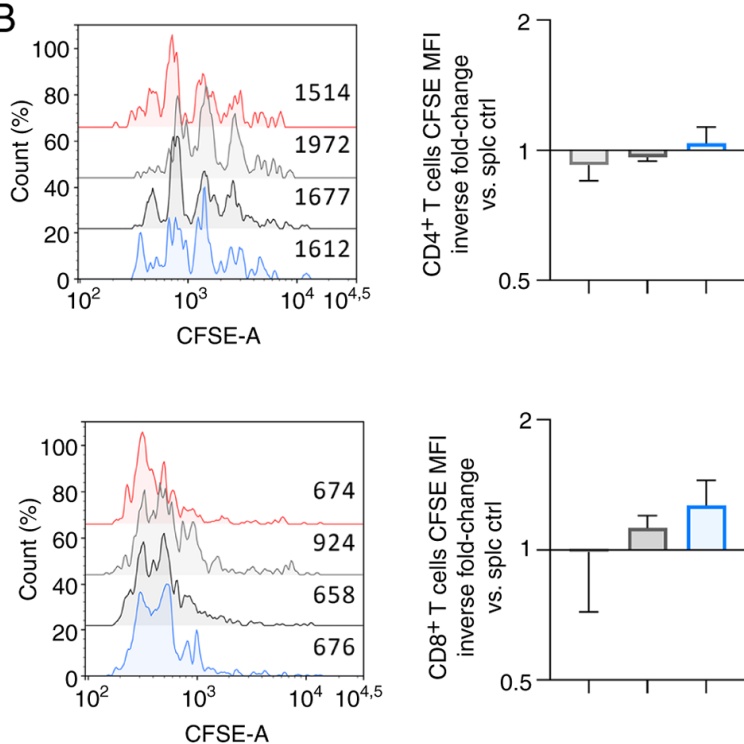
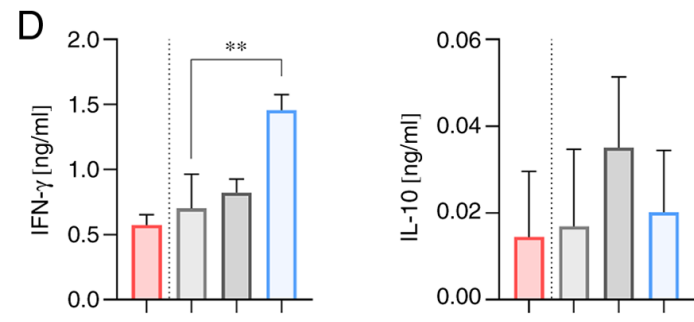

Figure 4. Influence of IL-10 silencing on the suppressive activity of PMN-MDSCs. CFSE-labeled splenocytes obtained from healthy mice were cultured with PMN-MDSCs (isolated from the in vitro culture of MDSCs using the FACS Aria sorter) at the final ratio of (A and C) 1:1 or (B and D) 2:1. (A and B) Proliferation of $\mathrm{CD}^{+}$and $\mathrm{CD}^{+}$spleen-derived $\mathrm{T}$ lymphocytes was measured by CFSE dilution and presented as fluorescence intensity on representative histograms. Splenocytes cultured with ConA and IL-2 (splc ctrl) were used as the positive control of proliferation. The results are presented as the mean inverse fold change of CFSE MFI calculated by reference to the splc ctrl group \pm SD [100\% splc ctrl MFI value-4818 and 2102 (A, CD4 ${ }^{+}$, exp. 1 and exp. 2 , respectively), 1662 and 796 (A, CD8 ${ }^{+}$, exp. 1 and exp. 2, respectively), 3813 and 1740 (B, CD4 ${ }^{+}$, exp. 1 and exp. 2, respectively), 1977 and 645 (B, CD8 ${ }^{+}$, exp. 1 and exp. 2 , respectively)]. (C and D) Concentrations of IFN- $\gamma$ and IL-10 in supernatants collected after $72 \mathrm{~h}$ co-culture of PMN-MDSCs with splenocytes measured using ELISA. The results are expressed as the mean \pm SD. Data obtained for two independent experiments measured in 1-3 repeats. Statistical significance was calculated using Welch's ANOVA followed by post hoc Dunnett's T3 multiple comparison test. Differences with a P-value $<0.05$ were regarded as significant ("* $\mathrm{P}<0.01)$. PMN-MDSC, polymorphonuclear myeloid-derived suppressor cells; MFI, mean fluorescence intensity.

in the splc ctrl group (Fig. 5K). Splenocytes co-cultured with myeloid cells isolated from the LV-treated groups according to two schemes of treatment secreted significantly higher amounts of IFN- $\gamma$ and IL-10 compared to those cultured in the presence of myeloid cells isolated from the untreated control (Fig. 5L and M). However, there were no considerable differences in production of these cytokines between shN LVs and shIL-10 LVs groups.

Analysis of lymphoid cells infiltrating tumors, performed according to the scheme presented in Fig. S7, showed that there was no significant influence of shIL-10 LV injections on the percentage of $\mathrm{CD}^{+} \mathrm{T}$ lymphocytes and NK cells in tumors compared to the untreated control (Fig. 6A). Nevertheless, LVs induced a high influx of $\mathrm{CD}^{+}$cells into tumors and there were visible differences in the percentage of these cells between the shN LV and shIL-10 LV groups. Namely, the percentage of the cells after injection of shN LVs was the highest on the 6th day and then slightly decreased, whereas in the group that received shIL-10 LVs the reaction was delayed and the highest percentage of $\mathrm{CD}^{+} \mathrm{T}$ lymphocytes was detected on the 10 th day and was considerably higher than in the untreated control and the shN LV group (Fig. 6A). In order to compare changes in the cytotoxic T lymphocytes (CTLs) and MDSC influx into tumors, the CTL/MDSCs ratios were calculated. The analysis demonstrated that the CTL/M-MDSC ratio of the shIL-10 LV group increased over time and on the 10th day was significantly higher than in the untreated control and the shN LV group, whereas the CTL/PMN-MDSC ratio was the lowest for the shIL-10 LV group on the 6th day and then increased to a similar level as in the other groups (Fig. 6B and C).

The analysis of $\mathrm{CD}^{+} \mathrm{T}$ lymphocytes in tumor-draining lymph nodes showed a significantly lower percentage of these cells in shN LV and shIL-10 LV groups than in the untreated control, which indicated an efflux of the cells from tLNs in the aftermath of LV injections (Figs. 6D and E, and S8). Although there were no significant differences in the percentage of CTLs between the shN and shIL-10 LV groups, on the 6th day the percentage of effector CTLs in the group receiving shIL-10 LVs was significantly higher than in the other groups. It showed that injections of LVs induced intensive mobilization 
A

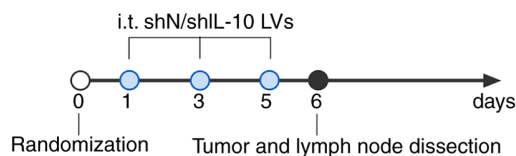

B

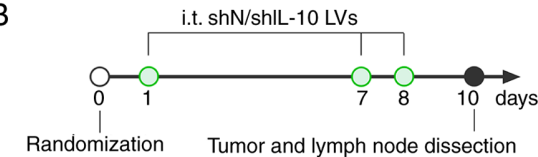

C

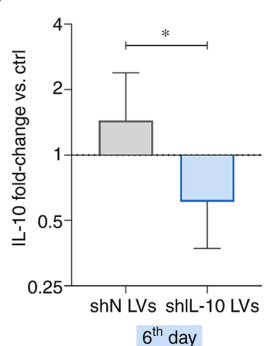

D

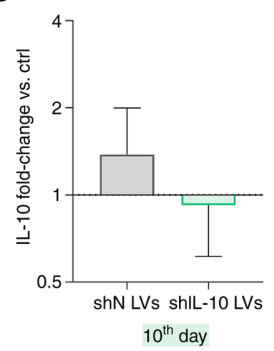

Myeloid cells in TME

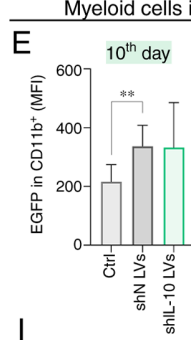

I

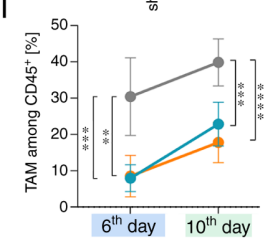

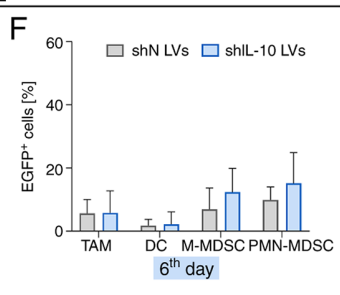
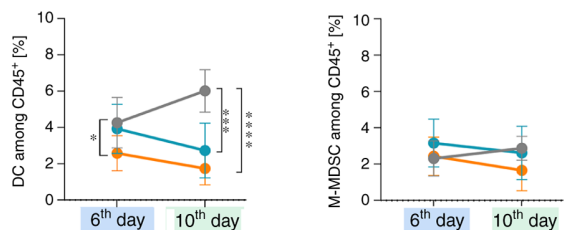

Ctrl

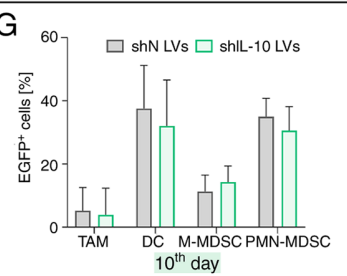

$\mathrm{H}$
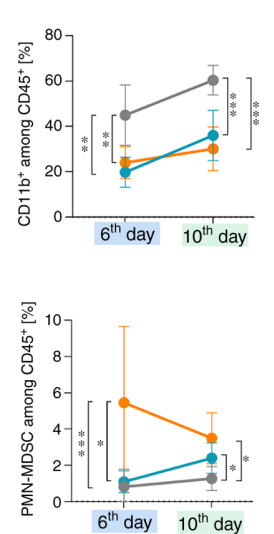

shlL-10 LVs
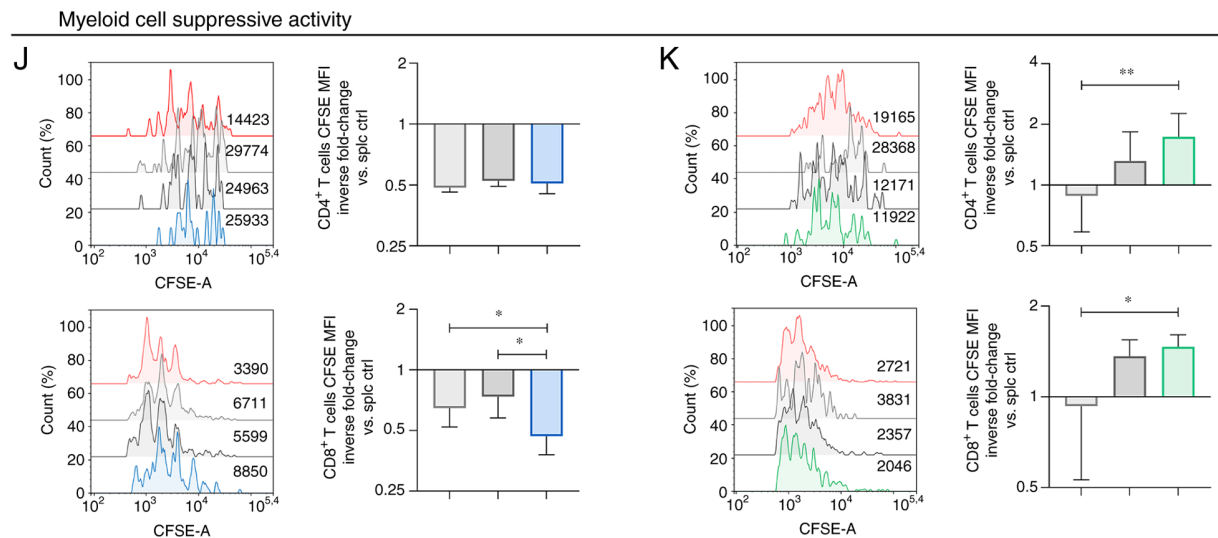

L
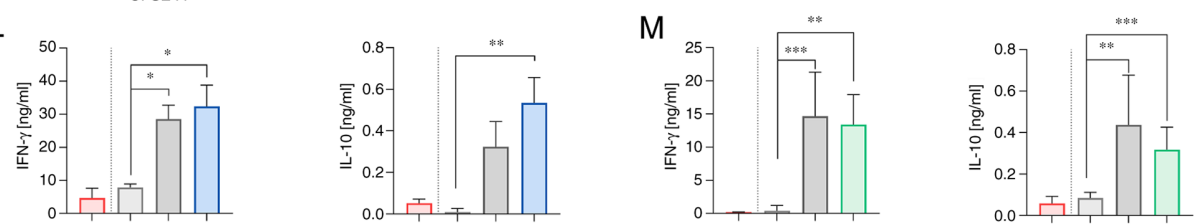

$$
\square_{\text {ctrl }}^{\text {Splc }} \quad \square \text { ctrl } \quad \square \text { shN LVs } \square \text { shll-10 LVs }
$$

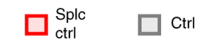

$\square$ shN LVs $\square$ shll-10 LVs

Figure 5. Influence of the intratumoral inoculation of IL-10 silencing lentivectors (shIL-10 LVs) on the myeloid cell activity. Tumors were dissected and analyzed on the 6th or 10th day after triple injection of LVs. (A and B) Schemes presenting administration schedules of shIL-10 LVs. (C and D) IL-10 production by cells isolated from tumors presented as the mean fold-change of IL-10 concentration in LV-treated groups in reference to the untreated group (ctrl) \pm SD measured using ELISA [100\% ctrl value-(C) $27.2 \mathrm{pg} / \mathrm{ml}$, (D) $231.6 \mathrm{pg} / \mathrm{ml}]$. (E) EGFP expression in myeloid cell infiltrating tumors on the 10 th day after the first LV injection. (F and $\mathrm{G})$ Changes in percentages of EGFP positive cells among selected subpopulations of myeloid cells on the 6th and 10th day, respectively. (H and I) Changes in percentages of myeloid cells and myeloid cell subpopulations in tumors on the 6th and the 10th days after LV inoculation estimated using multiparameter flow cytometry

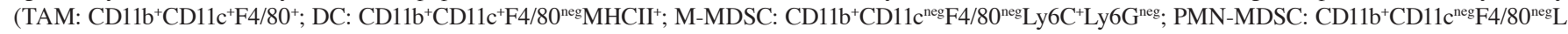
y6 $\left.\mathrm{C}^{\text {low }} \mathrm{Ly}_{6 \mathrm{G}^{+}}\right)$. ( $\mathrm{J}$ and $\left.\mathrm{K}\right)$ Suppressor activity of myeloid cells estimated using CFSE-based proliferation assay. The proliferation of $\mathrm{CD}^{+}{ }^{+}$and $\mathrm{CD}^{+}$spleen-derived $^{-}$ T lymphocytes was measured on the (J) 6th or on the (K) 10th day after LV injection. Splenocytes cultured with ConA and IL-2 (splc ctrl) were used as a positive control of proliferation. The results are presented as the mean inverse fold change of CFSE MFI calculated by reference to the splc ctrl group \pm SD [100\% splc ctrl MFI value-14509 and 4046 (J, CD4 ${ }^{+}$and CD8 ${ }^{+}$, respectively), 22382 and 3096 (K, CD4 ${ }^{+}$and CD8 ${ }^{+}$, respectively)]. (L and M) Concentrations of IFN- $\gamma$ and IL-10 in supernatants collected after $72 \mathrm{~h}$ co-culture of myeloid cells with splenocytes measured using ELISA. The results are expressed as the mean \pm SD. The number of mice per group in each experiment was 7-10. Statistical significance was calculated using (H-M) Welch's ANOVA followed by post hoc Dunnett's T3 multiple comparison test, (E) the non-parametric Kruskal-Wallis test followed by post hoc Dunn's multiple comparison test or (C and D) the Mann-Whitney U test. Differences with a $\mathrm{P}<0.05$ were regarded as significant $\left({ }^{*} \mathrm{P}<0.05,{ }^{* *} \mathrm{P}<0.01,{ }^{* * *} \mathrm{P}<0.001,{ }^{* * * *} \mathrm{P}<0.0001\right)$. shN LVs, lentiviral vectors encoding shN sequence; shIL-10 LVs, lentiviral vectors encoding shIL-10 sequence; TME, tumor microenvironment; MFI, mean fluorescent intensity; EGFP, enhanced green fluorescent protein; TAM, tumor-associated macrophages; DC, dendritic cells; M-MDSC, monocytic myeloid-derived suppressor cells; PMN-MDSC, polymorphonuclear myeloid-derived suppressor cells. 
Lymphoid cells in TME

A
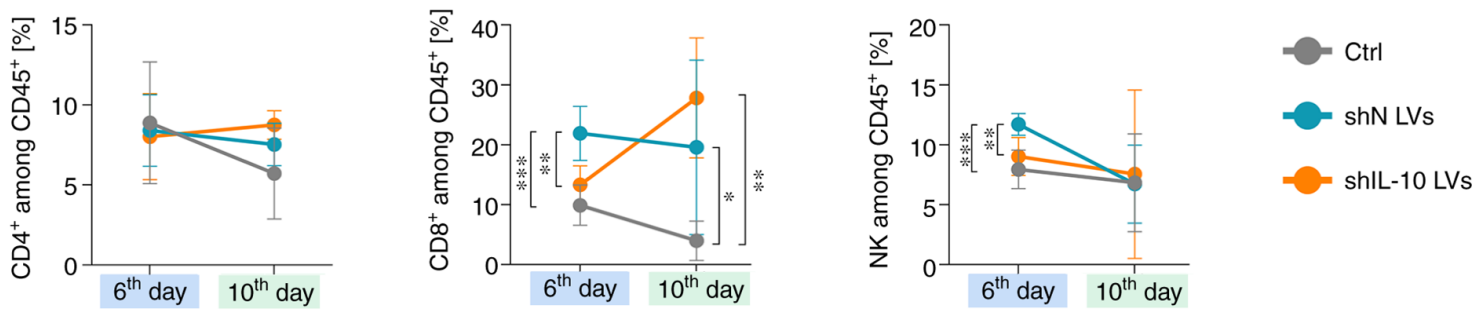

B

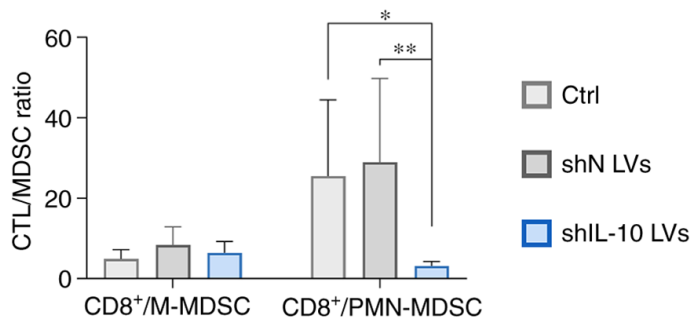

C

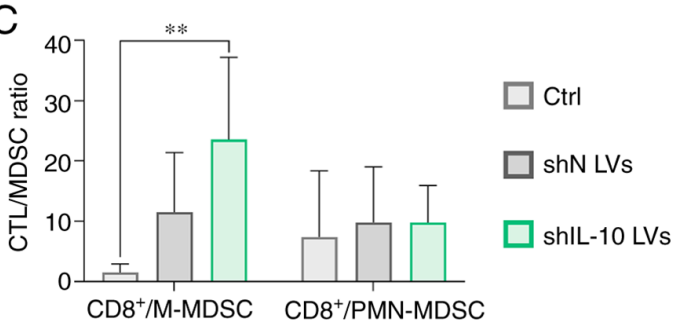

Cytotoxic T lymphocytes in tLN

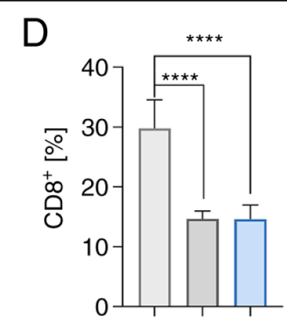

$\square$ Ctrl

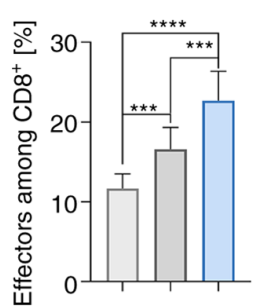

$\operatorname{shN} L \mathrm{~s}$

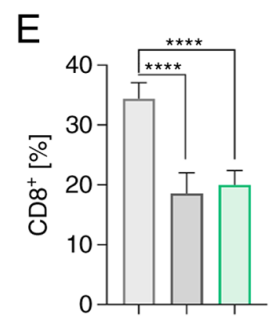

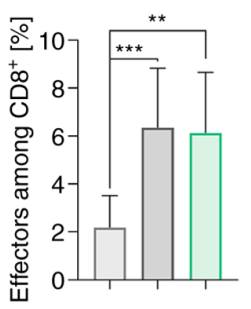

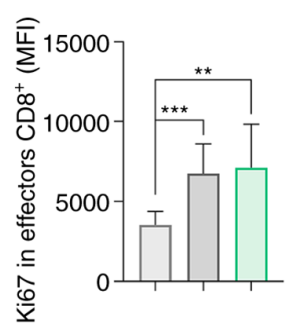

Ctrl

shN LVs

shIL-10 LVs

Figure 6. Influence of the intratumoral inoculation of IL-10 silencing lentivectors (shIL-10 LVs) on lymphoid cells in tumors and tumor-draining lymph nodes. Tumors and lymph nodes were dissected and analyzed using a multiparameter flow cytometry on the 6th or 10th day after triple injection of LVs. (A) Changes in percentages of $\mathrm{CD} 4^{+} \mathrm{T}$ lymphocytes $\left(\mathrm{CD} 3^{+} \mathrm{CD} 49 \mathrm{~b}^{\text {neg }} \mathrm{CD}^{+}\right)$, and $\mathrm{CD} 8^{+} \mathrm{T}$ lymphocytes $\left(\mathrm{CD} 3^{+} \mathrm{CD} 49 \mathrm{~b}^{\text {neg }} \mathrm{CD} 8^{+}\right)$, and NK cells $\left(\mathrm{CD} 3^{\text {neg }} \mathrm{CD} 49 \mathrm{~b}^{+}\right)$in tumors on the 6 th and the 10th days after LV inoculation. (B and C) The CTL to MDSC ratio calculated on the (B) 6 th and on the (C) 10th days after LV injection. (D) Changes in the percentage of CTLs and the percentage of effectors among them in tLNs on the 6th day after LV injection. (E) Changes in the percentage of CTLs, the percentage of effectors among them, and expression of Ki67 by effector CTLs in tLNs on the 10th day after LV injection. The results are expressed as the mean \pm SD. The number of mice per group in each experiment was 7-10. Statistical significance was calculated using (A, D and E) Welch's ANOVA followed by post hoc Dunnett's T3 multiple comparison test or (B and C) the non-parametric Kruskal-Wallis test followed by post hoc Dunn's multiple comparison test. Differences with a $\mathrm{P}<0.05$ were regarded as significant $\left({ }^{*} \mathrm{P}<0.05,{ }^{* *} \mathrm{P}<0.01,{ }^{* * *} \mathrm{P}<0.001,{ }^{* * * *} \mathrm{P}<0.0001\right)$. shN LVs, lentiviral vectors encoding shN sequence; shIL-10 LVs, lentiviral vectors encoding shIL-10 sequence; TME, tumor microenvironment; MFI, mean fluorescent intensity; M-MDSC, monocytic myeloid-derived suppressor cells; PMN-MDSC, polymorphonuclear myeloid-derived suppressor cells; CTL, cytotoxic T lymphocytes; tLN, tumor-draining lymph nodes.

of $\mathrm{CD}^{+}$cells in tLNs. However, shIL-10 LVs can be more effective in activation of effector CTLs.

In sum, the obtained data showed that intratumoral injections of shIL-10 LVs induced an increased influx of PMN-MDSCs and delayed infiltration of CD8 ${ }^{+} \mathrm{T}$ lymphocytes into the TME. Considering that shIL-10 LVs induced increased activation of effector CTLs in tLNs on the 6th day, one can assume that the lower percentage of $\mathrm{CD}^{+} \mathrm{T}$ lymphocytes in tumors in comparison to the shN LV-treated group results from the suppressive activity of PMN-MDSCs which infiltrated into the tumors in the aftermath of shIL-10 LV injections but have not been transduced yet. Prolonged observation revealed that on the 10th day, an enhanced percentage of shIL-10 LV-transduced MDSCs was correlated with a higher $\mathrm{T}$ lymphocyte proliferation rate in the presence of myeloid cells isolated from the shIL-10 LV group and an increased CTL/M-MDSC ratio in comparison to the untreated group. It may indicate that IL-10 downregulation in the tumor contributes to a reduction of immunosuppression, which depends on PMN-MDSC activity in the TME.

Pretreatment with cyclophosphamide reduced the effectiveness of LVs in transduction of myeloid cells in situ while simultaneously increasing their effect on mobilization of CTLs and NK cells into MC38 tumor. Due to the well-documented immunomodulatory activity of low doses of cyclophosphamide and its potential application in combination with different forms of immunotherapy we decided determine what the effect of pretreatment with CY on the activity of shIL-10 LVs in the TME would be. LVs were administered according to the aforementioned schemes; however, two days before their application mice received $\mathrm{CY}$ in a dose of $150 \mathrm{mg} / \mathrm{kg}$ body weight. Detailed schemes of the treatment are presented in Fig. 7A and B. Tumors, and tumor-draining 
lymph nodes were dissected on the 8th and 12th day after CY injection and since $\mathrm{CY}$ was administered two days prior to the first injection of LVs, the timepoints of analyses correspond to those in experiments performed without CY (6th and 10th day after LV injection). The obtained data showed that CY administered alone or with shN LVs slightly reduced the ability of cells isolated from tumor tissues to secrete IL-10 in comparison to the untreated group, which indicated the direct role of $\mathrm{CY}$ in reduction of the concentration of this cytokine in the TME. After application of CY + shIL-10 LVs the effect of specific IL-10 silencing was observed, but the difference between CY and CY + shIL-10 LV groups was statistically significant only on the 12th day (Fig. 7C and D). Furthermore, the EGFP analysis demonstrated that the mean intensity of EGFP fluorescence in CD11 ${ }^{+}$myeloid cells from CY + LVs groups was at the same level as in the CY group (on the 8th day) or the untreated control (on the 12th day) (Fig. 7E). On the other hand, an increased intensity of EGFP fluorescence was observed in tumor cells and lymphoid cells (Fig. S5C and D). It may be concluded that myeloid cells were less susceptible to LVs in the TME due to the differentiating activity of CY. Results confirming this hypothesis are presented in Fig. S9.

Nevertheless, the combined application of CY and shIL-10 LVs induced significant changes in the myeloid cell influx into tumors. Although the application of CY contributed to the decrease of CD11b cells in tumors, which was noticed on both the 8th and 12th day after treatment starting, LV injections enhanced the effect, and the percentage of myeloid cells in the shIL-10 LV group on the 12th day was significantly lower than in the untreated control and the CY group. The differences between shN and shIL-10 LV groups were rather small (Fig. 7F). Detailed analysis of myeloid cell subpopulations revealed that the application of CY with LVs (both $\mathrm{shN}$ and shIL-10) led to almost complete elimination of TAMs and DCs from the TME (Fig. 7G), while the percentage of M-MDSCs and PMN-MDSCs increased after application of CY + LVs (Fig. 7G). In spite of the increased proportion of MDSCs in tumors after application of CY $+\mathrm{LVs}$, the CFSE-based proliferation assay showed that although suppressor activity of myeloid cells isolated from the tumor was inconsiderably lower than the one detected for the untreated group on the 8th day, proliferation rates of lymphocytes in these groups were still lower than in the splc control group (Fig. 7H). Additionally, it was observed that myeloid cells isolated from tumors of the $\mathrm{CY}+$ shIL-10 LV group on the 12th day showed the highest suppressive activity toward $\mathrm{CD}^{+} \mathrm{T}$ lymphocytes (Fig. 7I), while the opposite effect was observed in the case of $\mathrm{CD}^{+} \mathrm{T}$ lymphocytes (Fig. 7I). The influence of the treatment on proliferation of spleen-derived lymphocytes was reflected in cytokine production by splenocytes co-cultured with myeloid cells. Data presented in Fig. 7J indicate a strong influence of $\mathrm{CY}$ on the activity of myeloid cells isolated from tumors on the 8th day. However, on the 12th day, CY was losing its activity and a large increase in the production of IFN- $\gamma$, as well as IL-10 was detected in the groups receiving $\mathrm{CY}+\mathrm{LVs}$ (Fig. 7K).

Analysis of lymphoid cells in tumors showed a strong influence of the combined treatment on the CTL and NK cell influx into the TME. Cyclophosphamide induced changes in the percentage of $\mathrm{CD}^{+} \mathrm{T}$ lymphocytes and on the 8 th day proportions of those cells were higher in the CY and CY + LV groups compared to the untreated control. However, on the 12th day a large influx of CTLs was observed only after combined treatment (Fig. 8A). On the 12th day, a significant increase of the percentage of NK cells in tumors was also observed following the CY $+\mathrm{LV}$ treatment (Fig. 8A), while no differences were noted between shN LV and shIL-10 LV groups. It was also demonstrated that the CTL/M-MDSC ratio was slightly more favorable for the CY + shIL-10 LV group than for the CY + shN LV group both on the 8th and 12th day, whereas the CTL/PMN-MDSC ratio was considerably lower for both CY + LV groups compared to the control groups on the 8th day and increased over time to the control level (Fig. 8B and C).

On the other hand, the analysis of $\mathrm{CD}^{+} \mathrm{T}$ lymphocytes in tumor-draining lymph nodes indicated that pretreatment with CY delayed the mobilization of the cells and on the 8th day there were no significant changes in the percentage and activity of CTLs in tLNs (Fig. 8D), while on the 12th day the efflux of CTLs, as well as a significant increase in the percentage of effector CTLs and a high proliferation index of these cells, was observed after application of the combined treatment. A slightly higher percentage of effector CTLs in the shIL-10 LV group compared to the shN LV group (Fig. 8E) was also observed.

In summary, CY induced the reduction of IL-10 in the TME by itself, so differences in IL-10 concentration in the TME could be insufficient to detect changes in proportions and activity of cells infiltrating tumors treated with CY + LVs. However, we observed that after the combined treatment, tumor cells and lymphoid cells were the major targets for intratumorally administered LVs, and such treatment activated not only $\mathrm{CD}^{+} \mathrm{T}$ lymphocytes but also NK cells.

\section{Discussion}

The main objective of this work was to determine the mechanisms of the immune response induced by lentiviral vectors encoding the shRNA sequence that silence the expression of IL-10. Taking into considerations our previous observations, we focused on evaluation of suppressor activity of MDSCs following treatment with shIL-10 LVs. For this purpose, shIL-10 LVs were used for transduction of ex vivo-generated MDSCs. During the second stage of this research, lentiviral vectors were injected into MC38 colon carcinoma tumors in order to evaluate the changes in the local immune response caused by shIL-10 LV-mediated reduction of IL-10 in the TME.

In the first stage of our research, it was observed that silencing of IL-10 in MDSCs, which were generated from bone marrow cells cultured in the presence of MC38 colon carcinoma cell-derived factors, did not induce their differentiation towards mature myeloid cell populations. To test whether IL-10 silencing affected MDSC activity, the effects of MDSC/shIL-10 on $\mathrm{T}$ cell proliferation and their ability to produce IFN- $\gamma$ and IL-10 were determined. We decided to examine M-MDSCs and PMN-MDSCs separately, since these two subpopulations of MDSCs use different suppression mechanisms. According to the literature, M-MDSCs suppress $\mathrm{T}$ cell activity by secretion of nitrogen oxide, arginase and 
A

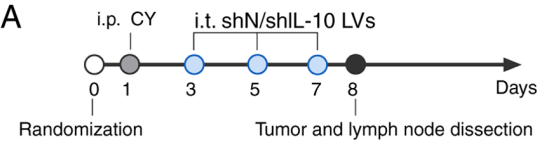

B

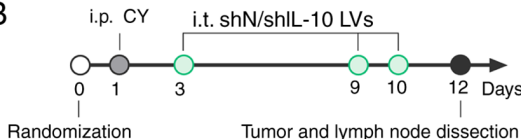

C

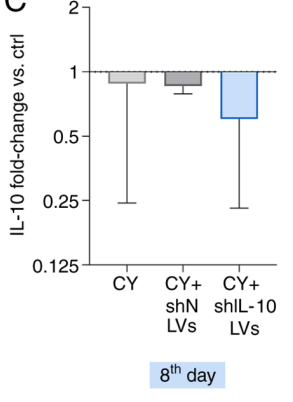

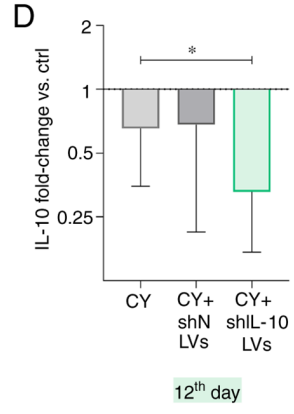

Myeloid cells in TME

$\mathrm{E}$

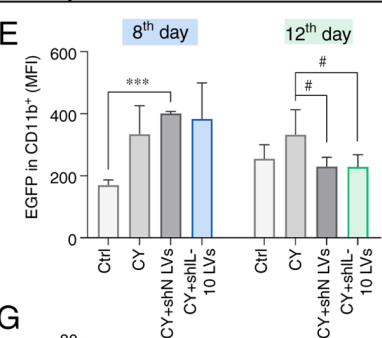

$\mathrm{F}$
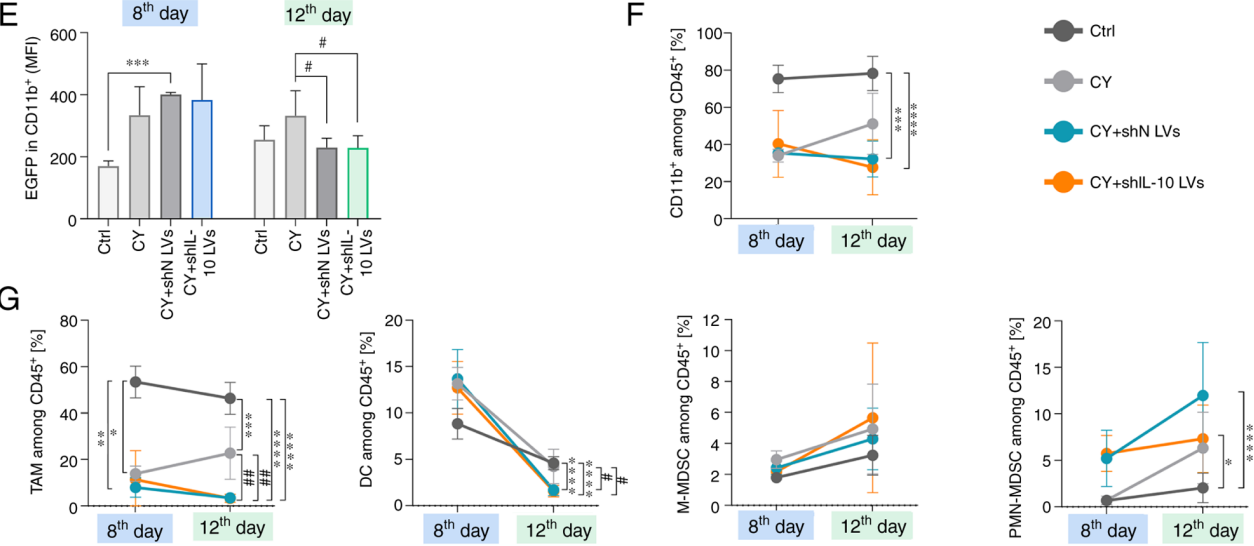

Myeloid cell suppressive activity

$\mathrm{H}$
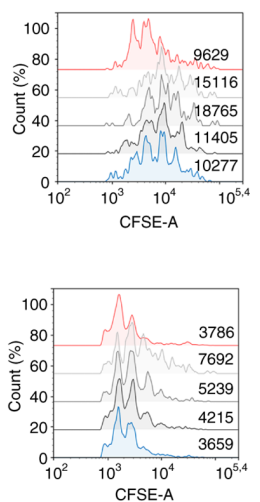

J

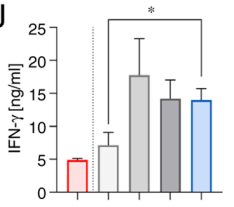

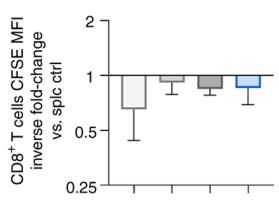
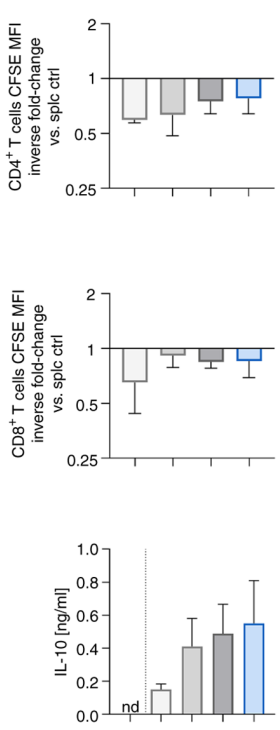
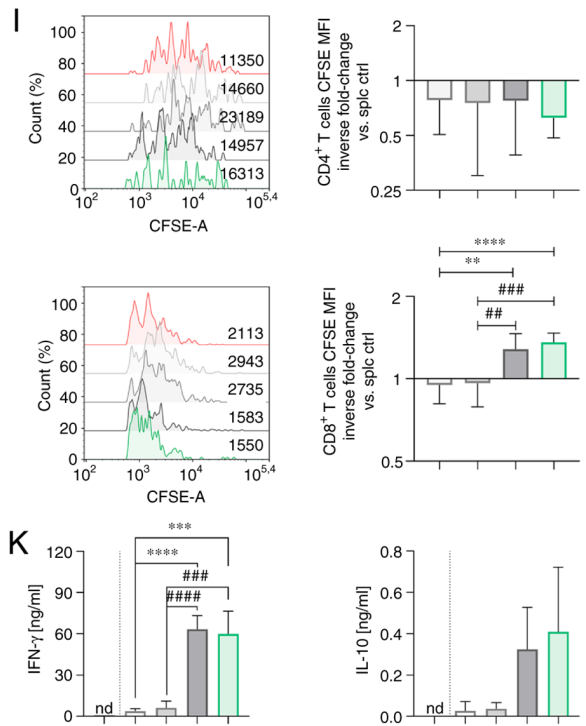
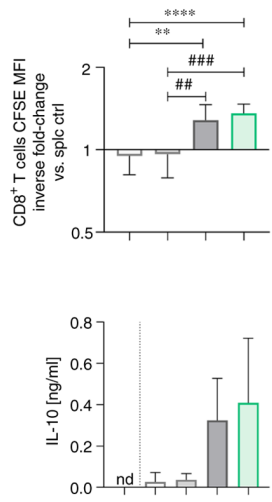

$\square_{\mathrm{ctrl}}^{\mathrm{Splc}}$

$\square \mathrm{ctrl} \quad \square \mathrm{cr}$

$\square \mathrm{CY}+$ shN LVs $\square \mathrm{CY}+$ shlL-10 LVs

$\square=$

$\square$ Ctrl

CY

CY+shN LVs $\square \mathrm{CY}+$ shlL-10 LVs

Figure 7. Myeloid cell activity in MC38 tumor in aftermath of shIL-10 LV injections preceded by administration of cyclophosphamide. Tumors were dissected and analyzed on the 8th or 12th day after intraperitoneal administration of CY followed by triple injection of LVs. (A and B) Schemes presenting the CY and LV administration schedules. (C and D) IL-10 production by cells isolated from tumors presented as the mean fold change of IL-10 concentration in CY + LV-treated groups referenced by the untreated group (ctrl) \pm SD measured using ELISA [100\% ctrl value-(C) 317 pg/ml and (D) 393 pg/ml]. (E) EGFP expression in myeloid cell infiltrating tumors. ( $\mathrm{F}$ and $\mathrm{G}$ ) Changes in percentages of myeloid cells and myeloid cell subpopulations in tumors on the 8th and the 12th day after treatment

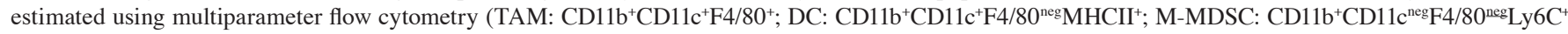

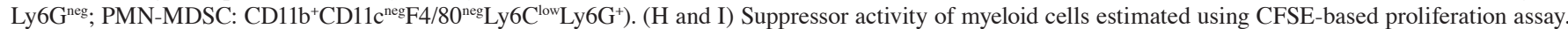
Proliferation of $\mathrm{CD}^{+}$and $\mathrm{CD}^{+}$spleen-derived T lymphocytes was measured on the (H) 8th or on the (I) 12th day after treatment. Splenocytes cultured with ConA and IL-2 (splc ctrl) were used as a positive control of proliferation. The results are presented as the mean inverse fold change of CFSE MFI calculated by reference to the splc ctrl group \pm SD [100\% splc ctrl MFI value-9400 and 3767 (H, CD4 ${ }^{+}$and CD8 ${ }^{+}$, respectively), 11950 and 2239 (I, CD4 ${ }^{+}$and CD8 ${ }^{+}$, respectively)]. ( $\mathrm{J}$ and $\mathrm{K}$ ) Concentrations of IFN- $\gamma$ and IL-10 in supernatants collected after $72 \mathrm{~h}$ co-culture of myeloid cells with splenocytes measured using ELISA (nd, not detected). The results are expressed as the mean \pm SD. The number of mice per group in experiment A was 3 and in experiment B 9-10. Statistical significance was calculated using Welch's ANOVA followed by post hoc Dunnett's T3 multiple comparison test. Differences with a P-value $<0.05$ were regarded as significant

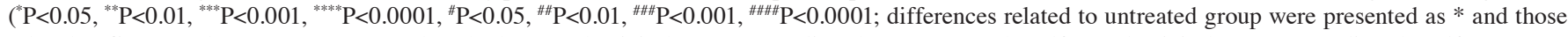
related to CY-treated group were presented as \#). shN LVs, lentiviral vectors encoding shN sequence; shIL-10 LVs, lentiviral vectors encoding shIL-10 sequence; CY, cyclophosphamide; TME, tumor microenvironment; MFI, mean fluorescent intensity; EGFP, enhanced green fluorescent protein; TAM, tumor-associated macrophages; DC, dendritic cells; M-MDSC, monocytic myeloid-derived suppressor cells; PMN-MDSC, polymorphonuclear myeloid-derived suppressor cells. 
A

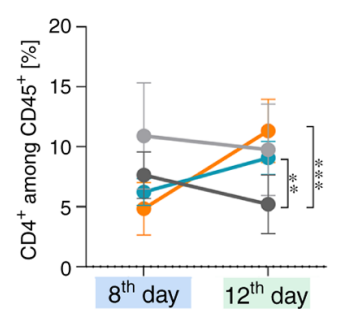

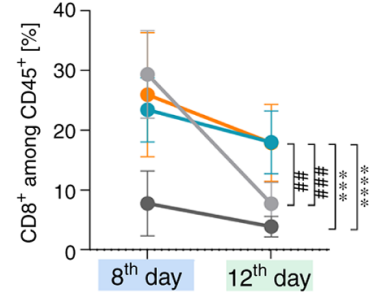

B

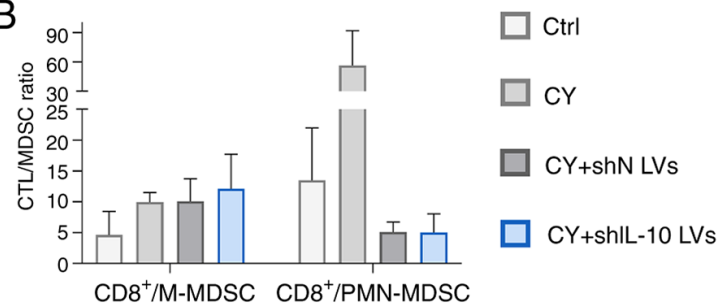

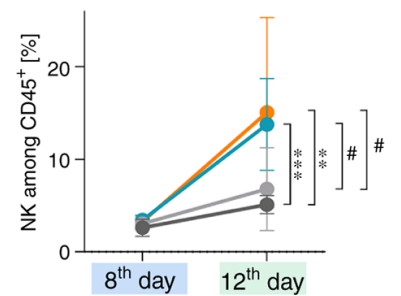

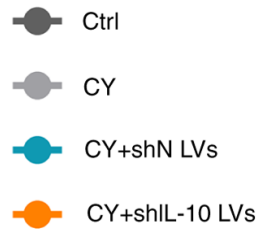

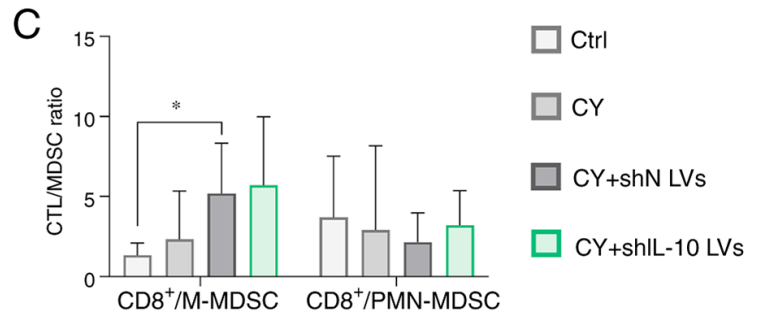

Cytotoxic T lymphocytes in tLN

D

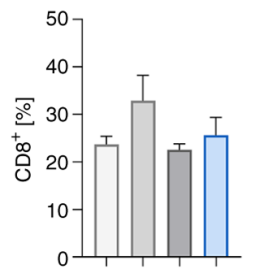

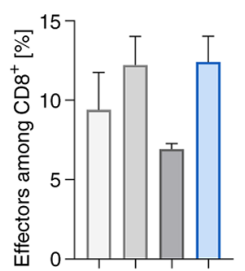

E

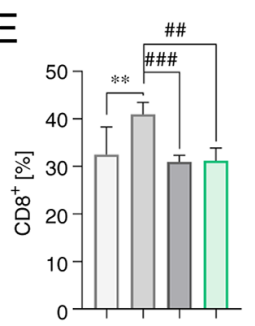

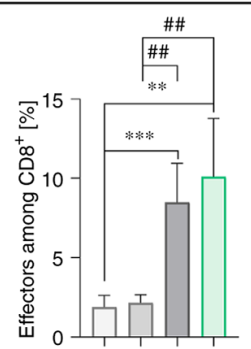

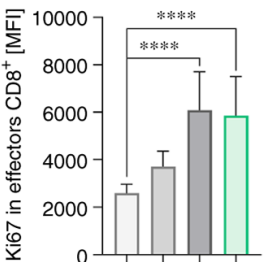

$\square$ Ctrl $\square$ CY $\square$ CY+shN LVs $\square$ CY+shlL-10 LVs

$\square$ Ctrl

$\square \mathrm{CY} \quad \square \mathrm{CY}+\mathrm{shN} L \mathrm{ss}$

$\square \mathrm{CY}+\mathrm{shlL}-10 \mathrm{LVs}$

Figure 8. Lymphoid cell activity in MC38 tumor and tumor-draining lymph nodes in aftermath of shIL-10 LV injections preceded by administration of cyclophosphamide. Tumors and tLNs were dissected and analyzed using flow cytometry on the 8th or 12th day after intraperitoneal administration of CY

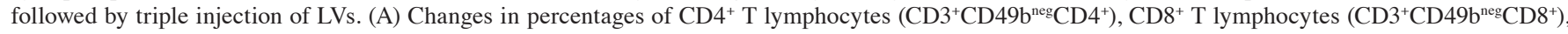
and NK cells $\left(\mathrm{CD}^{\mathrm{neg}} \mathrm{CD} 49 \mathrm{~b}^{+}\right)$in tumors on the 8 th and the 12 th days after treatment. (B and C) CTL to MDSC ratio calculated on the (B) 8th and on the (C) 12th days after treatment. (D) Changes in the percentage of CTLs and effectors among them in tLNs on the 8th day after treatment. (E) Changes in the percentage of CTLs, effectors among them, and expression of Ki67 by effector CTLs in tLNs on the 12th day after treatment. The results are expressed as the mean \pm SD. The number of mice per group in experiment A was 3 and in experiment B 9-10. Statistical significance was calculated using (A and D) Welch's ANOVA followed by post hoc Dunnett's T3 multiple comparison test or the (B, C and E) non-parametric Kruskal-Wallis test followed by post hoc Dunn's multiple comparison test. Differences with a $\mathrm{P}<0.05$ were regarded as significant $\left({ }^{*} \mathrm{P}<0.05,{ }^{* * *} \mathrm{P}<0.01,{ }^{* * * *} \mathrm{P}<0.001,{ }^{* * * *} \mathrm{P}<0.0001,{ }^{\#} \mathrm{P}<0.05,{ }^{\# \#} \mathrm{P}<0.01\right.$,

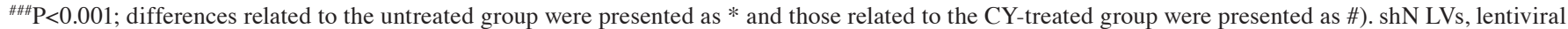
vectors encoding shN sequence; shIL-10 LVs, lentiviral vectors encoding shIL-10 sequence; CY, cyclophosphamide; TME, tumor microenvironment; MFI, mean fluorescent intensity; M-MDSC, monocytic myeloid-derived suppressor cells; PMN-MDSC, polymorphonuclear myeloid-derived suppressor cells; CTL, cytotoxic T lymphocytes; tLN, tumor-draining lymph nodes.

immunosuppressive cytokines, whereas PMN-MDSCs do so mostly by the production of reactive oxygen and nitrogen species, and due to the low stability of these compounds, they affect lymphocytes during cell-to-cell contact (3). During our research, it was observed that $\mathrm{T}$ cells proliferated less intensively in the presence of M-MDSC/shIL-10 than in cultures with non-transduced cells. Moreover, M-MDSC/shIL-10 decreased the ability of lymphocytes to produce IFN- $\gamma$ and IL-10. On the other hand, it seems that lentiviral vector-mediated silencing of IL-10 in PMN-MDSCs decreased their suppressor activity against $\mathrm{T}$ lymphocytes - these cells divided more often and also secreted more IFN- $\gamma$ and less IL-10 than lymphocytes cultured in the presence of non-transduced PMN-MDSCs. It should be noted that the suppressor activity of MDSC/shIL-10 did not differ significantly from MDSC/shN. The changes of the activity of MDSCs transduced with shN LVs are probably a result of the lentiviral vector influence on these cells. Since LVs contain viral antigens, as well as viral RNA, they modulate the activity of myeloid cells by toll-like receptor stimulation (22). Consequently, transduction of MDSCs with LVs may result in increased expression of molecules responsible for the activity of myeloid cells, e.g. MHC II, CD86, PD-L1, as well as in a changed cytokine expression profile. Taking into consideration the fact that MDSCs transduced with shN LVs showed a lower level of IL-10 expression than control cells, but still significantly higher than MDSC/shIL-10, it can be noted that the effect of MDSC transduction on their suppressive activity correlated with the 
efficiency of reduction of IL-10 expression. Nevertheless, the obtained data suggest that IL-10 silencing may result in completely opposite changes in the activity of M-MDSCs and PMN-MDSCs. In the literature there are few reports on the effect of IL-10 on MDSC activity, and the recent publications present divergent conclusions. Tanikawa et al (23) conducted studies using IL-10 knockout mice. During tumor development in IL-10 $10^{-/}$mice, they observed higher accumulation of MDSCs than in wild-type mice. This effect was also correlated with increased suppressor activity of IL-10 $1{ }^{-/}$MDSCs. However, research conducted by Noman et al (24) showed that MDSC-mediated suppression can be partially abolished by IL-10 neutralization, since after adding anti-IL-10 antibodies to MDSC and T cell cultures, the percentage of $\mathrm{CD} 8^{+} \mathrm{IFN}-\gamma^{+}$ and $\mathrm{CD} 4^{+} \mathrm{IFN}-\gamma^{+}$cells increased (24). It should be emphasized that in the mentioned papers, MDSCs were identified as

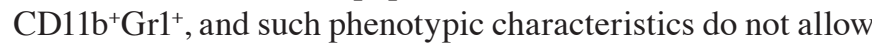
separation of the two subpopulations of cells. Considering our results described above, it is possible that the observations regarding the effect of IL-10 on MDSC activity depend on the proportion between monocytic and polymorphonuclear cell subpopulations. However, to confirm this assumption, further research is needed to elucidate the influence of IL-10 on the individual mechanisms used by MDSCs.

Interleukin-10, occurring in high concentrations in the TME, can be produced by MDSCs, but also by other myeloid cell populations, such as TAMs and tolerogenic DCs, as well as by tumor-infiltrating lymphocytes, e.g. Tregs and Th2 lymphocytes. In some cases, IL-10 is also secreted directly by tumor cells (16). Since MC38 murine colon carcinoma cells do not produce IL-10 in vitro, it can be assumed that the main source of this cytokine in MC38 tumors is immune cells. In order to reduce the concentration of IL-10 in TME, lentiviral vectors encoding shRNA sequences against IL-10 were injected into MC38 tumors. The advantage of the lentiviral vectors is their ability to transduce a wide spectrum of cells, both proliferating and resting (25). After intratumoral administration of lentiviral vectors, they can therefore transduce tumor cells and immune cells present in the TME. The transduced cells can proliferate and accumulate at the injection site for up to three weeks after subcutaneous administration of the vectors (26). On the other hand, antiviral immune response elicited after LV injections may lead to the elimination of transduced cells from the TME by antigen-specific CTLs (22). There is a constant effort to minimize the limitations of the lentiviral vector system resulting from low efficiency and immunogenicity $(27,28)$. Hence, research aimed at determining the mechanisms triggered by the in vivo application of lentiviral vectors is of key importance in the development of LV-based therapies.

Taking this into consideration, in this work, lentiviral vectors were administered three times according to two schedules. The first one assumed intratumoral LV injections every two days and the end of the experiment on the 6th day from the start of the study. Since adaptive antiviral response takes about one week to develop (29), at this timepoint, an immune response after the use of LVs may be observed, but the elimination of transduced cells should not occur yet. In the second scheme, the interval between the first and second administrations of LVs was extended to 6 days, in order to check whether repeated administration of the vectors after the development of the adaptive immune response induced by the first injection would also be effective in reducing the concentration of IL-10 in the TME, which was measured on the 10th day after the first LV application. Additionally, performing the experiments in such a way enabled us to determine the kinetics of changes occurring in the activity of cells of the immune system due to lentiviral vector-mediated immunomodulation, as well as due to the decrease in the concentration of IL-10 in the TME resulting from transduction of cells with shRNA specific for IL-10.

Analysis of expression of EGFP in the TME, as well as IL-10 production by cells isolated from tumors confirmed that intratumorally injected LVs were able to transduce predominantly myeloid cells inside tumors and deliver sequences encoding shRNA specific for IL-10, and, as a result, reduce the concentration of IL-10 in the TME. Due to a low number of leukocytes in the MC38 TME, we were not able to analyze the IL-10 secretion by each myeloid cell population, but the percentage of $\mathrm{EGFP}^{+}$cells demonstrates the efficiency of transduction of individual populations. The main recipients of lentiviral transduction were PMN-MDSCs, as observed in both schemes of the treatment. However, on the 10th day after LV inoculations, the percentage of EGFP ${ }^{+}$ cells among PMN-MDSCs was almost two-fold higher than on the 6th day. This effect may be related to the influx of PMN-MDSCs enhanced by LV injections. A similar response to viral application was observed by Tan et al (30) during oncolytic virotherapy of mesothelioma. In this case, recruitment of IL-10-secreting PMN-MDSCs into the TME resulted in inhibition of DC-mediated induction of CTLs. Therefore, it can be assumed that elevated suppressor activity of myeloid cells isolated from tumors on the 6th day of the treatment with shIL-10 LVs is related to the insufficient efficacy of transduction and, as a result, high expression of IL-10 in these cells. In contrast, analyses performed on the 10th day after LV inoculations revealed that myeloid cells isolated from shIL-10 LV-treated tumors did not suppress the activity of $\mathrm{T}$ cells. We suppose that this effect corresponded to the higher percentage of transduced PMN-MDSCs, which was presumably observed as a result of the recruitment of these cells into the TME after the first injection of LVs and their in-situ transduction by LVs administered six days later. The loss of suppressor activity of myeloid cells isolated from tumors injected with shIL-10 LVs is in line with the results obtained during analysis of ex vivo-generated PMN-MDSCs transduced with shIL-10 LVs.

Since the analyses presented in this work were performed on the 6th and 10th day after LV administration, the observation was too short to determine the changes in tumor growth rate induced by LVs. However, our previously published research demonstrated that triple injection of shIL-10 LVs carried out at weekly intervals resulted in significant MC38 tumor growth inhibition, which was $71.5 \%$ in relation to the untreated control on the 23rd day of treatment (17). Another study of ours aimed to determine the influence of IL-10 elimination, mediated by anti-IL-10 antibodies (Ab), on the effectiveness of cyclophosphamide and dendritic cell-based therapy (31). We observed that temporary systemic reduction of IL-10 did not lead to tumor growth inhibition but resulted in 
decreased suppressive activity of splenic-derived MDSCs. The changes induced by anti-IL-10 Ab led to enhanced efficiency of dendritic cell-based therapy.

Cyclophosphamide is known for its immunomodulatory properties and contribution to the enhancement of the anti-tumor response induced during immunotherapy. Since our previous research proved that it augments the antitumor response of shIL-10 LVs (17), schemes of tumor inoculations with LVs were extended by single i.p. CY administration prior to the LV injections. It was proved that $\mathrm{CY}$ used in low doses causes the selective elimination of regulatory $\mathrm{T}$ lymphocytes, and by inducing immunogenic death of cancer cells, at the same time contributes to the increase in the activity of effector $\mathrm{T}$ lymphocytes and supports the formation of immune memory (32). As a result of the changes of the immune landscape mediated by CY, a decrease of IL-10 expression is often observed (33-35). In fact, production of IL-10 by cells isolated from tumors was decreased not only in CY and shIL-10 LV-treated groups, but a slight decrease was also observed in groups treated with $\mathrm{CY}$ as well as CY and shN LVs. Analysis of EGFP expression in the TME revealed that pretreatment with $\mathrm{CY}$ changed the susceptibility of cells to transduction-in this case only CD $45^{\text {neg }}$ and lymphoid cell transduction was observed. Lack of transduction of myeloid cells was presumably related to the effect of cyclophosphamide on the maturation of myeloid cells. TAMs and DCs identified in the TME of cytostatic-treated mice showed an increased level of expression of MHC II and CD86 molecules compared to the untreated control. This could be caused by the accumulation of DAMPs in the TME, released by cancer cells during the immunogenic cell death induced by cyclophosphamide (36). The presence of these molecules in the TME causes an increased influx of dendritic cells, which absorb the released tumor antigens, and then migrate to the tumor-draining lymph nodes and activate antigen-specific $\mathrm{T}$ cells. Schiavoni et al (37) found that cyclophosphamide promotes the expansion of the cross-presentation dendritic cell population, resulting in stimulation of tumor antigen-specific cytotoxic T lymphocytes. This is in line with our observation that on the 8th day after $\mathrm{CY}$ administration an increase of the percentage of $\mathrm{CD}^{+} \mathrm{T}$ cells and, to a lesser extent, $\mathrm{CD}^{+} \mathrm{T}$ cells among leukocytes infiltrating tumors was correlated with an increase of the percentage of effector cells among $\mathrm{CD}^{+} \mathrm{T}$ cells identified in tumor-draining lymph nodes. The effect of CY on the CTL population was only temporary and on the 12th day after its administration was not observed. However, modulation of the TME by CY led to elicitation of a CTL- and NK-dependent response induced by LVs on the 12th day of the treatment. There are reports in the literature indicating that cyclophosphamide may increase the accumulation of MDSCs, which reduces its therapeutic effect $(38,39)$. However, the present research did not confirm these observations-myeloid cells isolated from the CY-treated group did not show higher $\mathrm{T}$ cell suppressor activity than cells from untreated tumors. Despite the lack of myeloid cell transduction after treatment with CY and LVs, on the 12th day suppressor activity of these cells towards $\mathrm{CD} 8^{+} \mathrm{T}$ cells was significantly decreased. However, presumably due to the decrease of IL-10 secretion in the TME mediated by CY, we did not observe any differences between LV-treated groups.
Additionally, since after pretreatment with CY we did not note that LV transduced myeloid cells inside tumors, no effect of shIL-10 LV-mediated reduction of IL-10 production by myeloid cells could be observed.

In conclusion, the obtained data may indicate that downregulation of IL-10 expression with lentiviral vectors encoding shRNA against IL-10 in ex-vivo-generated, as well as tumor-derived MDSCs results in modulation of their suppressor activity. However, this effect seems to be dependent not only on the silencing of IL-10 expression but also on the transduction with lentiviral vectors. Hence, to confirm this assumption, further studies on the effects of IL-10 depletion on the suppressor mechanisms used by these cells are required. Such knowledge would make it possible to establish novel therapeutic regimens, which could become a foundation for future strategies in antitumor treatment of patients with a high frequency of MDSCs.

\section{Acknowledgements}

Not applicable.

\section{Funding}

This research was funded by National Science Centre, Poland (grant nos. 2014/15/N/NZ4/04817 and 2018/30/E/NZ5/00711).

\section{Availability of data and materials}

The datasets used and/or analyzed during the current study are available from the corresponding authors on reasonable request.

\section{Authors' contributions}

NAG and JR were involved in conceptualization. NAG, JR and EPP developed the methodology. NAG and JR performed formal analysis. NAG, KWC, AS, JM, EPP and JR performed the experiments. NAG and JR prepared the original draft. KWC, AS, JM and EPP reviewed and edited the manuscript. JR supervised the study. NAG and JR acquired funding. NAG and JR confirm the authenticity of all the raw data. All authors read and approved the final manuscript.

\section{Ethics approval and consent to participate}

All experiments were performed in accordance with Directive 2010/63/EU of the European Parliament and of the Council on the protection of animals used for scientific purposes and were approved by the 1st Local Ethic Committee for Experiments with the Use of Laboratory Animals, Wrocław, Poland (authorization no. 11/2015, 33/2018).

\section{Patient consent for publication}

Not applicable.

\section{Competing interests}

The authors declare that they have no competing interests. 


\section{References}

1. Buoncervello M, Gabriele L and Toschi E: The janus face of tumor microenvironment targeted by immunotherapy. Int J Mol Sci 20: 4320, 2019.

2. Pitt JM, Marabelle A, Eggermont A, Soria JC, Kroemer G and Zitvogel L: Targeting the tumor microenvironment: Removing obstruction to anticancer immune responses and immunotherapy. Ann Oncol 27: 1482-1492, 2016.

3. Kumar V, Patel S, Tcyganov E and Gabrilovich DI: The nature of myeloid-derived suppressor cells in the tumor microenvironment. Trends Immunol 37: 208-220, 2016.

4. Marvel D and Gabrilovich DI: Myeloid-derived suppressor cells in the tumor microenvironment: Expect the unexpected. J Clin Invest 125: 3356-3364, 2015.

5. Gnjatic S, Bronte V, Brunet LR, Butler MO, Disis ML, Galon J, Hakansson LG, Hanks BA, Karanikas V, Khleif SN, et al: Identifying baseline immune-related biomarkers to predict clinical outcome of immunotherapy. J Immunother Cancer 5: 44, 2017.

6. Spencer KR, Wang J, Silk AW, Ganesan S, Kaufman HL and Mehnert JM: Biomarkers for immunotherapy: Current developments and challenges. Am Soc Clin Oncol Educ Book 35: e493-e503, 2016.

7. Masucci GV, Cesano A, Hawtin R, Janetzki S, Zhang J, Kirsch I, Dobbin KK, Alvarez J, Robbins PB, Selvan SR, et al: Validation of biomarkers to predict response to immunotherapy in cancer: Volume I-pre-analytical and analytical validation. J Immunother Cancer 4: 76, 2016.

8. Anger N and Rossowska J: Myeloid-derived suppressor cells as a target for anticancer therapy. Postępy Higieny i Medycyny Doświadczalnej 72: 1179-1198, 2018.

9. Xiu B, Lin Y, Grote DM, Ziesmer SC, Gustafson MP, Maas ML, Zhang Z, Dietz AB, Porrata LF, Novak AJ, et al: IL-10 induces the development of immunosuppressive CD14(+)HLA-DR(low/-) monocytes in B-cell non-Hodgkin lymphoma. Blood Cancer J 5 : e328, 2015

10. Wu L, Deng Z, Peng Y, Han L, Liu J, Wang L, Li B, Zhao J, Jiao $\mathrm{S}$ and Wei H: Ascites-derived IL-6 and IL-10 synergistically expand $\mathrm{CD} 14^{+} \mathrm{HLA}-\mathrm{DR}^{-/ \mathrm{low}}$ myeloid-derived suppressor cells in ovarian cancer patients. Oncotarget 8: 76843-76856, 2017.

11. Steinbrink K, Wölfl M, Jonuleit H, Knop J and Enk AH: Induction of tolerance by IL-10-treated dendritic cells. J Immunol 159 : $4772-4780,1997$

12. Cavani A, Nasorri F, Prezzi C, Sebastiani S, Albanesi C and Girolomoni G: Human $\mathrm{CD}^{+} \mathrm{T}$ lymphocytes with remarkable regulatory functions on dendritic cells and nickel-specific Th1 immune responses. J Invest Dermatol 114: 295-302, 2000.

13. Sica A, Saccani A, Bottazzi B, Polentarutti N, Vecchi A, van Damme J and Mantovani A: Autocrine production of IL-10 mediates defective IL-12 production and NF-kappa B activation in tumor-associated macrophages. J Immunol 164: 762-767, 2000.

14. Dennis KL, Blatner NR, Gounari F and Khazaie K: Current status of interleukin-10 and regulatory T-cells in cancer. Curr Opin Oncol 25: 637-645, 2013

15. Fiorentino DF, Bond MW and Mosmann TR: Two types of mouse T helper cell. IV. Th2 clones secrete a factor that inhibits cytokine production by Th1 clones. J Exp Med 170: 2081-2095, 1989.

16. Sato T, Terai M, Tamura Y, Alexeev V, Mastrangelo MJ and Selvan SR: Interleukin 10 in the tumor microenvironment: A target for anticancer immunotherapy. Immunol Res 51: 170-182, 2011.

17. Rossowska J, Anger N, Szczygieł A, Mierzejewska J and Pajtasz-PiaseckaE: Reprogramming the murinecolon cancermicroenvironment using lentivectors encoding shRNA against IL-10 as a component of a potent DC-based chemoimmunotherapy. J Exp Clin Cancer Res 37: 126, 2018

18. Sistigu A, Viaud S, Chaput N, Bracci L, Proietti E and Zitvogel L: Immunomodulatory effects of cyclophosphamide and implementations for vaccine design. Semin Immunopathol 33: 369-383, 2011.

19. Pajtasz-Piasecka E, Szyda A, Rossowska J, Krawczenko A, Indrová M, Grabarczyk P, Wysocki P, Mackiewicz A and Duś D: Loss of tumorigenicity of murine colon carcinoma MC38/0 cell line after transduction with a retroviral vector carrying murine IL-12 genes. Folia Biol (Praha) 50: 7-14, 2004.

20. Rossowska J, Pajtasz-Piasecka E, Anger N, Wojas-Turek J, Kicielińska J, Piasecki E and Duś D: Cyclophosphamide and IL-12-transduced DCs enhance the antitumor activity of tumor antigen-stimulated DCs and reduce Tregs and MDSCs number J Immunother 37: 427-439, 2014
21. Rossowska J, Anger N, Szczygieł A, Mierzejewska J and Pajtasz-Piasecka E: Intratumoral lentivector-mediated TGF- $\beta 1$ gene downregulation as a potent strategy for enhancing the antitumor effect of therapy composed of cyclophosphamide and dendritic cells. Front Immunol 8: 713, 2017.

22. Breckpot K, Escors D, Arce F, Lopes L, Karwacz K, Van Lint S, Keyaerts M and Collins M: HIV-1 Lentiviral vector immunogenicity is mediated by toll-like receptor 3 (TLR3) and TLR7. J Virol 84: 5627-5636, 2010.

23. Tanikawa T, Wilke CM, Kryczek I, Chen GY, Kao J, Núñez G and Zou W: Interleukin (IL)-10 ablation promotes tumor development, growth and metastasis. Cancer Res 72: 420-429, 2012.

24. Noman MZ, Desantis G, Janji B, Hasmim M, Karray S, Dessen P, Bronte V and Chouaib S: PD-L1 is a novel direct target of HIF-1 $\alpha$, and its blockade under hypoxia enhanced MDSC-mediated T cell activation. J Exp Med 211: 781-790, 2014.

25. Breckpot K, Emeagi PU and Thielemans K: Lentiviral vectors for anti-tumor immunotherapy. Curr Gene Ther 8: 438-448, 2008.

26. Furmanov K, Elnekave M, Lehmann D, Clausen BE, Kotton DN and Hovav AH: The role of skin-derived dendritic cells in CD8+ T cell priming following immunization with lentivectors. J Immunol 184: 4889-4897, 2010.

27. Olbrich H, Slabik C and Stripecke R: Reconstructing the immune system with lentiviral vectors. Virus Genes 53: 723-732, 2017.

28. Milone MC and O'Doherty U: Clinical use of lentiviral vectors. Leukemia 32: 1529-1541, 2018

29. Nash AA and Dutia BM: The Immune response to viral infections. In: Topley \& Wilson's Microbiology and Microbial Infections. American Cancer Society, 15 Mar, 2010 doi: 10.1002/9780470688618.taw0220.

30. Tan Z, Liu L, Chiu MS, Cheung KW, Yan CW, Yu Z, Lee BK, Liu W, Man K and Chen Z: Virotherapy-recruited PMN-MDSC infiltration of mesothelioma blocks antitumor CTL by IL-10-mediated dendritic cell suppression. Oncoimmunology 8: e1518672, 2019.

31. Rossowska J, Anger N, Kicielińska J, Pajtasz-Piasecka E, Bielawska-Pohl A, Wojas-Turek J and Duś D: Temporary elimination of IL-10 enhanced the effectiveness of cyclophosphamide and BMDC-based therapy by decrease of the suppressor activity of MDSCs and activation of antitumour immune response. Immunobiology 220: 389-398, 2015.

32. Abu Eid R, Razavi GSE, Mkrtichyan M, Janik J and Khleif SN: Old-school chemotherapy in immunotherapeutic combination in cancer, a low-cost drug repurposed. Cancer Immunol Res 4: 377-382, 2016

33. Huang XM, Zhang NR, Lin XT, Zhu CY, Zou YF, Wu XJ, He XS, He XW, Wan YL and Lan P: Antitumor immunity of low-dose cyclophosphamide: Changes in T cells and cytokines TGF-beta and IL-10 in mice with colon-cancer liver metastasis. Gastroenterol Rep (Oxf) 8: 56-65, 2019.

34. Matar P, Rozados VR, Gervasoni SI and Scharovsky OG: Down regulation of T-cell-derived IL-10 production by low-dose cyclophosphamide treatment in tumor-bearing rats restores in vitro normal lymphoproliferative response. Int Immunopharmacol 1:307-319, 2001.

35. Xia Q, Geng F, Zhang FF, Liu CL, Xu P, Lu ZZ, Zhang HH, Kong W and Yu XH: Enhancement of fibroblast activation protein $\alpha$-based vaccines and adenovirus boost immunity by cyclophosphamide through inhibiting IL-10 expression in 4T1 tumor bearing mice. Vaccine 34: 4526-4535, 2016.

36. Vanmeerbeek I, Sprooten J, De Ruysscher D, Tejpar S, Vandenberghe P, Fucikova J, Spisek R, Zitvogel L, Kroemer G, Galluzzi L and Garg AD: Trial watch: Chemotherapy-induced immunogenic cell death in immuno-oncology. Oncoimmunology 9: 1703449, 2020 .

37. Schiavoni G, Sistigu A, Valentini M, Mattei F, Sestili P, Spadaro F, Sanchez M, Lorenzi S, D'Urso MT, Belardelli F, et al: Cyclophosphamide synergizes with type I interferons through systemic dendritic cell reactivation and induction of immunogenic tumor apoptosis. Cancer Res 71: 768-778, 2011.

38. Mikyšková R, Indrová M, Vlková V, Bieblová J, Šímová J, Paračková Z, Pajtasz-Piasecka E, Rossowska J and Reiniš M: DNA demethylating agent 5-azacytidine inhibits myeloid-derived suppressor cells induced by tumor growth and cyclophosphamide treatment. J Leukoc Biol 95: 743-753, 2014

39. Sevko A, Sade-Feldman M, Kanterman J, Michels T, Falk CS, Umansky L, Ramacher M, Kato M, Schadendorf D, Baniyash M and Umansky V: Cyclophosphamide promotes chronic inflammation-dependent immunosuppression and prevents antitumor response in melanoma. J Invest Dermatol 133: 1610-1619, 2013.

This work is licensed under a Creative Commons Attribution 4.0 International (CC BY-NC 4.0) License 